\title{
Reliability Modelling and Analysis of Redundant Systems Connected to Supporting External Device for Operation Attended by a Repairman and Repairable Service Station
}

\author{
Ibrahim Yusuf', Rabia Salihu Said ${ }^{2}$, Fatima Salman Koki², Mansur Babagana ${ }^{3}$ \\ ${ }^{1}$ Department of Mathematical Sciences, Bayero University, Kano, Nigeria \\ ${ }^{2}$ Department of Physics, Bayero University, Kano, Nigeria \\ ${ }^{3}$ Department of Computer Science and Information Technology, Bayero University, Kano, Nigeria \\ Email: iyusuf.mth@buk.edu.ng, rssaidu.phy@buk.edu.ng, FatimaSK2775@gmail.com, \\ mbabagana.cs@buk.edu.ng
}

Received 5 October 2014; revised 1 November 2014; accepted 24 November 2014

Copyright (C) 2014 by authors and Scientific Research Publishing Inc.

This work is licensed under the Creative Commons Attribution International License (CC BY).

http://creativecommons.org/licenses/by/4.0/

(c) (i) Open Access

\begin{abstract}
In this paper, probabilistic models for three redundant configurations have been developed to analyze and compare some reliability characteristics. Each system is connected to a repairable supporting external device for operation. Repairable service station is provided for immediate repair of failed unit. Explicit expressions for mean time to system failure and steady-state availability for the three configurations are developed. Furthermore, we compare the three configurations based on their reliability characteristics and found that configuration II is more reliable and efficient than the remaining configurations.
\end{abstract}

\section{Keywords}

Availability, Supporting Device, Service Station, Redundancy

\section{Introduction}

High system reliability and availability play a vital role towards industrial growth as the profit is directly dependent on production volume which depends upon system performance. Thus the reliability and availability of a system may be enhanced by proper design, optimization at the design stage and by maintaining the same dur- 
ing its service life. Because of their prevalence in power plants, manufacturing systems, and industrial systems, many researchers have studied reliability and availability problem of different systems (see, for instance, Ref [1]-[8] and the references therein). In real-life situations we often encounter cases where the systems that cannot work without the help of external supporting devices connect to such systems. These external supporting devices are systems themselves that are bound to fail. Where such systems exist, a repairable service station is provided for the immediate repair of failed unit. Such systems are found in power plants, manufacturing systems, and industrial systems. Ref [9] [10] performed comparative analysis of some reliability characteristics between redundant systems requiring supporting units for their operation.

The problem considered in this paper is different from the work of Ref [9] [10]. The objectives of the present paper are three. The first is to develop the explicit expressions for the mean time to system failure (MTSF) and steadystate availability. The second objective is to perform a parametric investigation of various system parameters on mean time to system failure (MTSF) and steady-state availability and capture their impact on the mean time to system failure (MTSF) and steady-state availability. The third objective is to perform comparative analysis between the three configurations based on assumed numerical values in order to determine the optimal configuration.

\section{Description of the Systems}

We consider three redundant systems connected to an external supporting device for their operation as follows. The first system is a 2-out-of-3 system connected to a supporting device and has a repairable service station. The second is also a 2-out-of-3 system connected to supporting device and has two standby repairable service stations. The third system is a 3-out-of-4 system connected to a supporting device and has a repairable service station. We assume that switching is perfect and instantaneous. We also assume that two units cannot fail simultaneously. Whenever a unit fails with failure rate $\beta_{1}$, it is immediately sent to a service station for repair with service rate $\alpha_{1}$. However, on the course of repairing failed unit, the service is bound to fail with failure rate of $\beta_{2}$ and service rate of $\alpha_{2}$ and failed unit must wait whenever the service station is under repair for first and third system, while the standby service will continue repairing failed unit for the second system. The supporting device is a system that is prone to failure. Whenever the supporting device failed with rate $\beta_{3}$ it is attended by a repairman, the system stop working and must wait until the supporting device is repaired with rate $\alpha_{3}$.

\section{Mean Time to System Failure Models Formulation}

\subsection{MTSF Formulation for Configuration I}

For configuration I, we define $P_{i}(t)$ to be the probability that the system at time $t \geq 0$ is in state $S_{i}$. Also let $P(t)$ be the probability row vector at time $t$, we have the following initial condition:

$$
P(0)=\left[P_{0}(0), P_{1}(0), P_{2}(0), P_{3}(0), P_{4}(0), P_{5}(0), P_{6}(0), P_{7}(0)\right]=[1,0,0,0,0,0,0,0]
$$

We obtain the following differential equations:

$$
\begin{aligned}
& \frac{\mathrm{d} P_{0}(t)}{\mathrm{d} t}=-\left(\beta_{1}+\beta_{2}\right) P_{0}(t)+\alpha_{1} P_{1}(t)+\alpha_{2} P_{2}(t) \\
& \frac{\mathrm{d} P_{1}(t)}{\mathrm{d} t}=-\left(\alpha_{1}+\sum_{k=1}^{3} \beta_{k}\right) P_{1}(t)+\beta_{1} P_{0}(t)+\alpha_{3} P_{3}(t)+\alpha_{1} P_{4}(t)+\alpha_{2} P_{5}(t) \\
& \frac{\mathrm{d} P_{2}(t)}{\mathrm{d} t}=-\alpha_{2} P_{2}(t)+\beta_{2} P_{0}(t) \\
& \frac{\mathrm{d} P_{3}(t)}{\mathrm{d} t}=-\left(\alpha_{3}+\beta_{1}+\beta_{2}\right) P_{3}(t)+\beta_{3} P_{1}(t)+\alpha_{2} P_{7}(t) \\
& \frac{\mathrm{d} P_{4}(t)}{\mathrm{d} t}=-\left(\alpha_{1}+\beta_{3}\right) P_{4}(t)+\beta_{1} P_{1}(t)+\alpha_{3} P_{6}(t) \\
& \frac{\mathrm{d} P_{5}(t)}{\mathrm{d} t}=-\left(\alpha_{2}+\beta_{3}\right) P_{5}(t)+\beta_{2} P_{1}(t)+\alpha_{3} P_{7}(t) \\
& \frac{\mathrm{d} P_{6}(t)}{\mathrm{d} t}=-\alpha_{3} P_{6}(t)+\beta_{1} P_{3}(t)+\beta_{3} P_{4}(t) \\
& \frac{\mathrm{d} P_{7}(t)}{\mathrm{d} t}=-\left(\alpha_{2}+\alpha_{3}\right) P_{7}(t)+\beta_{2} P_{3}(t)+\beta_{3} P_{5}(t)
\end{aligned}
$$


This can be written in the matrix form as

$$
\dot{P}=T_{1} P
$$

where

$$
T_{1}=\left[\begin{array}{cccccccc}
-\left(\beta_{1}+\beta_{2}\right) & \alpha_{1} & \alpha_{2} & 0 & 0 & 0 & 0 & 0 \\
\beta_{1} & -\left(\alpha_{1}+\sum_{k=1}^{3} \beta_{k}\right) & 0 & \alpha_{3} & \alpha_{1} & \alpha_{2} & 0 & 0 \\
\beta_{2} & 0 & -\alpha_{2} & 0 & 0 & 0 & 0 & 0 \\
0 & \beta_{3} & 0 & -\left(\alpha_{3}+\beta_{1}+\beta_{2}\right) & 0 & 0 & 0 & \alpha_{2} \\
0 & \beta_{1} & 0 & 0 & -\left(\alpha_{1}+\beta_{3}\right) & 0 & \alpha_{3} & 0 \\
0 & \beta_{2} & 0 & 0 & 0 & -\left(\alpha_{2}+\beta_{3}\right) & 0 & \alpha_{3} \\
0 & 0 & 0 & \beta_{1} & \beta_{3} & 0 & -\alpha_{3} & 0 \\
0 & 0 & 0 & \beta_{2} & 0 & \beta_{3} & 0 & -\left(\alpha_{2}+\alpha_{3}\right)
\end{array}\right]
$$

It is difficult to evaluate the transient solutions, the procedure to develop the explicit expression for $M_{T S F_{1}}$ is to delete the rows and column of absorbing states of matrix $T_{1}$ and take the transpose to produce a new matrix, say $M_{1}$. Following Ref [11] [12], the expected time to reach an absorbing state is obtained from

$$
E\left[T_{P(0) \rightarrow P(\text { absorbing })}\right]=M T S F_{1}=P(0)\left(-Q_{1}^{-1}\right)\left[\begin{array}{l}
1 \\
1 \\
1
\end{array}\right]=\frac{N_{0}}{D_{0}}
$$

where

$$
\begin{aligned}
& Q_{1}=\left[\begin{array}{ccc}
-\left(\beta_{1}+\beta_{2}\right) & \beta_{1} & 0 \\
\alpha_{1} & -\left(\alpha_{1}+\sum_{k=1}^{3} \beta_{k}\right) & \beta_{3} \\
0 & \alpha_{3} & -\left(\alpha_{3}+\beta_{1}+\beta_{2}\right)
\end{array}\right] \\
& N_{0}=\alpha_{1}\left(\alpha_{3}+\beta_{1}+\beta_{2}\right)+2 \beta_{1}\left(\alpha_{3}+\beta_{1}+2 \beta_{2}+\beta_{3}\right)+\beta_{2}\left(\alpha_{3}+\beta_{2}+\beta_{3}\right) \\
& D_{0}=\alpha_{1} \beta_{1} \beta_{2}+\alpha_{3} \beta_{1}^{2}+\beta_{1}^{3}+3 \beta_{1}^{2} \beta_{2}+2 \alpha_{3} \beta_{1} \beta_{2}+3 \beta_{1} \beta_{2}^{2}+\beta_{1}^{2} \beta_{3}+2 \beta_{1} \beta_{2} \beta_{3}+\alpha_{1} \alpha_{3} \beta_{2}+\alpha_{1} \beta_{2}^{2}+\alpha_{3} \beta_{2}^{2}+\beta_{2}^{3}+\beta_{2}^{2} \beta_{3}
\end{aligned}
$$

\subsection{MTSF Formulation for Configuration II}

For configuration II, we define $P_{i}(t)$ to be the probability that the system at time $t \geq 0$ is in state $S_{i}$. Also let $P(t)$ be the probability row vector at time $t$, we have the following initial condition:

$$
P(0)=\left[P_{0}(0), P_{1}(0), P_{2}(0), P_{3}(0), P_{4}(0), \cdots, P_{10}(0)\right]=[1,0,0,0,0,0,0,0,0,0,0]
$$

The differential equations are expressed in the form

$$
\dot{P}=T_{2} P
$$

where

$$
T_{2}=\left[\begin{array}{ccccccccccc}
-\left(\beta_{1}+\beta_{2}\right) & \alpha_{1} & \alpha_{2} & 0 & 0 & 0 & 0 & 0 & 0 & 0 & 0 \\
\beta_{1} & -y_{1} & 0 & \alpha_{3} & \alpha_{1} & \alpha_{2} & 0 & 0 & 0 & 0 & 0 \\
\beta_{2} & 0 & -\alpha_{2} & 0 & 0 & 0 & 0 & 0 & 0 & 0 & 0 \\
0 & \beta_{3} & 0 & -y_{2} & 0 & 0 & \alpha_{2} & \alpha_{1} & \alpha_{3} & 0 & 0 \\
0 & \beta_{1} & 0 & 0 & -y_{3} & 0 & 0 & \alpha_{3} & 0 & 0 & 0 \\
0 & \beta_{2} & 0 & 0 & 0 & -\alpha_{2} & 0 & 0 & 0 & 0 & 0 \\
0 & 0 & 0 & \beta_{2} & 0 & 0 & -\alpha_{2} & 0 & 0 & 0 & 0 \\
0 & 0 & 0 & \beta_{1} & \beta_{3} & 0 & 0 & -y_{4} & 0 & \alpha_{3} & 0 \\
0 & 0 & 0 & \beta_{3} & 0 & 0 & 0 & 0 & -y_{5} & 0 & \alpha_{2} \\
0 & 0 & 0 & 0 & 0 & 0 & 0 & \beta_{3} & 0 & -\alpha_{3} & 0 \\
0 & 0 & 0 & 0 & 0 & 0 & 0 & 0 & \beta_{2} & 0 & -\alpha_{2}
\end{array}\right]
$$


and

$$
y_{1}=\left(\alpha_{1}+\beta_{1}+\beta_{2}+\beta_{3}\right), y_{2}=\left(\alpha_{3}+\beta_{1}+\beta_{2}+\beta_{3}\right), y_{3}=\left(\alpha_{1}+\beta_{3}\right), y_{4}=\left(\alpha_{1}+\alpha_{3}+\beta_{3}\right), y_{5}=\left(\alpha_{3}+\beta_{1}+\beta_{2}\right)
$$

Using the procedure described in Subsection 3.1, the expected time to reach an absorbing state is

$$
E\left[T_{P(0) \rightarrow P(\text { absorbing })}\right]=M T S F_{2}=P(0)\left(-Q_{2}^{-1}\right)\left[\begin{array}{l}
1 \\
1 \\
1 \\
1
\end{array}\right]=\frac{N_{1}}{D_{1}}
$$

where

$$
\begin{aligned}
Q_{2}= & {\left[\begin{array}{cccc}
-\left(\beta_{1}+\beta_{2}\right) & \beta_{1} & 0 & 0 \\
\alpha_{1} & -\left(\alpha_{1}+\beta_{1}+\beta_{2}+\beta_{3}\right) & \beta_{3} & 0 \\
0 & \alpha_{3} & -\left(\alpha_{3}+\beta_{1}+\beta_{2}+\beta_{3}\right) & \beta_{3} \\
0 & 0 & \alpha_{3} & -\left(\alpha_{3}+\beta_{1}+\beta_{2}\right)
\end{array}\right] } \\
N_{1}= & 4 \beta_{1} \beta_{2} \beta_{3}+2 \alpha_{1} \alpha_{3} \beta_{1}+2 \alpha_{1} \alpha_{3} \beta_{2}+2 \alpha_{1} \beta_{1} \beta_{2}+\alpha_{1} \beta_{1} \beta_{3}+\alpha_{1} \beta_{2} \beta_{3}+4 \alpha_{3} \beta_{1} \beta_{2}+\alpha_{3} \beta_{1} \beta_{3} \\
& +\alpha_{3} \beta_{2} \beta_{3}+\alpha_{1} \alpha_{3}^{2}+\alpha_{1} \beta_{1}^{2}+\alpha_{1} \beta_{2}^{2}+\beta_{1}^{3}+\beta_{2}^{3}+\alpha_{3}^{2} \beta_{1}+2 \alpha_{3} \beta_{1}^{2}+3 \beta_{1}^{2} \beta_{2}+3 \beta_{1} \beta_{2}^{2} \\
& +2 \beta_{1}^{2} \beta_{3}+\alpha_{3}^{2} \beta_{2}+2 \alpha_{3} \beta_{2}^{2}+2 \beta_{2}^{2} \beta_{3}+\beta_{1} \beta_{3}^{2}+\beta_{2} \beta_{3}^{2}+\beta_{1}\left(\alpha_{3}^{2}+2 \alpha_{3} \beta_{1}+2 \alpha_{3} \beta_{2}\right. \\
& \left.+\beta_{1}^{2}+2 \beta_{1} \beta_{2}+\beta_{2}^{2}+\beta_{1} \beta_{3}+\beta_{2} \beta_{3}\right)+\beta_{1} \beta_{3}^{2}+\beta_{1} \beta_{3}\left(\alpha_{3}+\beta_{1}+\beta_{2}\right) \\
D_{1}= & 6 \beta_{1}^{2} \beta_{2} \beta_{3}+6 \beta_{1} \beta_{2}^{2} \beta_{3}+2 \alpha_{1} \alpha_{3} \beta_{1} \beta_{2}+\alpha_{1} \beta_{1} \beta_{2} \beta_{3}+2 \alpha_{3} \beta_{1} \beta_{2} \beta_{3}+4 \beta_{1} \beta_{2}^{3}+\alpha_{3}^{2} \beta_{1}^{2} \\
& +2 \alpha_{3} \beta_{1}^{3}+4 \beta_{1}^{3} \beta_{2}+6 \beta_{1}^{2} \beta_{2}^{2}+2 \beta_{1}^{3} \beta_{3}+\beta_{1}^{2} \beta_{3}^{2}+\alpha_{1} \beta_{2}^{3}+\alpha_{3}^{2} \beta_{2}^{2}+2 \alpha_{3} \beta_{2}^{3}+2 \beta_{2}^{3} \beta_{3} \\
& +\beta_{3}^{2} \beta_{2}^{2}+\alpha_{1} \beta_{1}^{2} \beta_{2}+6 \alpha_{3} \beta_{1}^{2} \beta_{2}+\alpha_{1} \beta_{1}^{2} \beta_{3}+2 \alpha_{1} \alpha_{3} \beta_{2}^{2}+\alpha_{1} \beta_{2}^{2} \beta_{3}+\alpha_{3} \beta_{2}^{2} \beta_{3} \\
& +2 \alpha_{1} \beta_{1} \beta_{2}^{2}+2 \alpha_{3}^{2} \beta_{1} \beta_{2}+6 \alpha_{1} \beta_{1} \beta_{2}^{2}+2 \beta_{1} \beta_{2} \beta_{3}^{2}+\alpha_{1} \alpha_{3}^{2} \beta_{2}+\beta_{1}^{4}+\beta_{2}^{4}
\end{aligned}
$$

\subsection{MTSF Formulation for Configuration III}

For configuration II, we define $P_{i}(t)$ to be the probability that the system at time $t \geq 0$ is in state $S_{i}$. Also let $P(t)$ be the probability row vector at time $t$, we have the following initial condition:

$$
P(0)=\left[P_{0}(0), P_{1}(0), P_{2}(0), P_{3}(0), P_{4}(0), P_{5}(0), P_{6}(0), P_{7}(0)\right]=[1,0,0,0,0,0,0,0]
$$

The differential equations are expressed in the form

$$
\dot{P}=T_{3} P
$$

where

$$
T_{3}=\left[\begin{array}{cccccccc}
-\left(\beta_{1}+\beta_{2}\right) & \alpha_{1} & \alpha_{2} & 0 & 0 & 0 & 0 & 0 \\
\beta_{1} & -\left(\alpha_{1}+\beta_{1}+\beta_{2}+\beta_{3}\right) & 0 & \alpha_{1} & \alpha_{2} & \alpha_{3} & 0 & 0 \\
\beta_{2} & 0 & -\alpha_{2} & 0 & 0 & 0 & 0 & 0 \\
0 & \beta_{1} & 0 & -\left(\alpha_{1}+\beta_{3}\right) & 0 & 0 & \alpha_{3} & 0 \\
0 & \beta_{2} & 0 & 0 & -\left(\alpha_{2}+\beta_{3}\right) & 0 & 0 & \alpha_{3} \\
0 & \beta_{3} & 0 & 0 & 0 & -\left(\alpha_{3}+\beta_{1}+\beta_{2}\right) & 0 & \alpha_{2} \\
0 & 0 & 0 & \beta_{3} & 0 & \beta_{1} & -\alpha_{3} & 0 \\
0 & 0 & 0 & 0 & \beta_{3} & \beta_{2} & 0 & -\left(\alpha_{2}+\alpha_{3}\right)
\end{array}\right]
$$

Using the procedure described in Subsection 3.1, the expected time to reach an absorbing state is 


$$
E\left[T_{P(0) \rightarrow P(\text { absorbing })}\right]=M T S F_{3}=P(0)\left(-Q_{3}^{-1}\right)\left[\begin{array}{l}
1 \\
1 \\
1
\end{array}\right]=\frac{N_{2}}{D_{2}}
$$

where

$$
\begin{gathered}
Q_{3}=\left[\begin{array}{ccc}
-\left(\beta_{1}+\beta_{2}\right) & \beta_{1} & 0 \\
\alpha_{1} & -\left(\alpha_{1}+\beta_{1}+\beta_{2}+\beta_{3}\right) & \beta_{3} \\
0 & \alpha_{3} & -\left(\alpha_{3}+\beta_{1}+\beta_{2}\right)
\end{array}\right] \\
N_{2}=\alpha_{1}\left(\alpha_{3}+\beta_{1}+\beta_{2}\right)+2 \beta_{1}\left(\alpha_{3}+\beta_{1}+2 \beta_{2}+\beta_{3}\right)+\beta_{2}\left(\alpha_{3}+\beta_{2}+\beta_{3}\right) \\
D_{2}= \\
\alpha_{1} \beta_{1} \beta_{2}+\alpha_{3} \beta_{1}^{2}+\beta_{1}^{3}+3 \beta_{1}^{2} \beta_{2}+2 \alpha_{3} \beta_{1} \beta_{2}+3 \beta_{1} \beta_{2}^{2}+\beta_{1}^{2} \beta_{3} \\
\\
+2 \beta_{1} \beta_{2} \beta_{3}+\alpha_{1} \alpha_{3} \beta_{2}+\alpha_{1} \beta_{2}^{2}+\alpha_{3} \beta_{2}^{2}+\beta_{2}^{3}+\beta_{2}^{2} \beta_{3}
\end{gathered}
$$

\section{Availability Models Formulation}

\subsection{Availability Model Formulation for Configuration I}

For the analysis of availability case of configuration I we use the same initial condition as in Subsection 3.1

$$
P(0)=\left[P_{0}(0), P_{1}(0), P_{2}(0), P_{3}(0), P_{4}(0), P_{5}(0), P_{6}(0), P_{7}(0)\right]=[1,0,0,0,0,0,0,0]
$$

The differential equations above are expressed in the form

$$
\left[\begin{array}{c}
\dot{P}_{0} \\
\dot{P}_{1} \\
\dot{P}_{2} \\
\dot{P}_{3} \\
\dot{P}_{4} \\
\dot{P}_{5} \\
\dot{P}_{6} \\
\dot{P}_{7}
\end{array}\right]=\left[\begin{array}{cccccccc}
-\left(\beta_{1}+\beta_{2}\right) & \alpha_{1} & \alpha_{2} & 0 & 0 & 0 & 0 & 0 \\
\beta_{1} & -\left(\alpha_{1}+\beta_{1}+\beta_{2}+\beta_{3}\right) & 0 & \alpha_{3} & \alpha_{1} & \alpha_{2} & 0 & 0 \\
\beta_{2} & 0 & -\alpha_{2} & 0 & 0 & 0 & 0 & 0 \\
0 & \beta_{3} & 0 & -\left(\alpha_{3}+\beta_{1}+\beta_{2}\right) & 0 & 0 & 0 & \alpha_{2} \\
0 & \beta_{1} & 0 & 0 & -\left(\alpha_{1}+\beta_{3}\right) & 0 & \alpha_{3} & 0 \\
0 & \beta_{2} & 0 & 0 & 0 & -\left(\alpha_{2}+\beta_{3}\right) & 0 & \alpha_{3} \\
0 & 0 & 0 & \beta_{1} & \beta_{3} & 0 & -\alpha_{3} & 0 \\
0 & 0 & 0 & \beta_{2} & 0 & \beta_{3} & 0 & -\left(\alpha_{2}+\alpha_{3}\right)
\end{array}\right]\left[\begin{array}{c}
P_{0} \\
P_{1} \\
P_{2} \\
P_{3} \\
P_{4} \\
P_{5} \\
P_{6} \\
P_{7}
\end{array}\right]
$$

The steady-state availability is given by

$$
A_{v 1}(\infty)=P_{0}(\infty)+P_{1}(\infty)+P_{3}(\infty)
$$

In the steady-state, the derivatives of the state probabilities become zero and therefore Equation (2) become

$$
T_{1} P=0
$$

which is in matrix form

$\left[\begin{array}{cccccccc}-\left(\beta_{1}+\beta_{2}\right) & \alpha_{1} & \alpha_{2} & 0 & 0 & 0 & 0 & 0 \\ \beta_{1} & -\left(\alpha_{1}+\beta_{1}+\beta_{2}+\beta_{3}\right) & 0 & \alpha_{3} & \alpha_{1} & \alpha_{2} & 0 & 0 \\ \beta_{2} & 0 & -\alpha_{2} & 0 & 0 & 0 & 0 & 0 \\ 0 & \beta_{3} & 0 & -\left(\alpha_{3}+\beta_{1}+\beta_{2}\right) & 0 & 0 & 0 & \alpha_{2} \\ 0 & \beta_{1} & 0 & 0 & -\left(\alpha_{1}+\beta_{3}\right) & 0 & \alpha_{3} & 0 \\ 0 & \beta_{2} & 0 & 0 & 0 & -\left(\alpha_{2}+\beta_{3}\right) & 0 & \alpha_{3} \\ 0 & 0 & 0 & \beta_{1} & \beta_{3} & 0 & -\alpha_{3} & 0 \\ 0 & 0 & 0 & \beta_{2} & 0 & \beta_{3} & 0 & -\left(\alpha_{2}+\alpha_{3}\right)\end{array}\right]\left[\begin{array}{l}P_{0}(\infty) \\ P_{1}(\infty) \\ P_{2}(\infty) \\ P_{3}(\infty) \\ P_{4}(\infty) \\ P_{5}(\infty) \\ P_{6}(\infty) \\ P_{7}(\infty)\end{array}\right]=\left[\begin{array}{l}0 \\ 0 \\ 0 \\ 0 \\ 0 \\ 0 \\ 0 \\ 0\end{array}\right]$

Using the following normalizing condition 


$$
\sum_{J=0}^{7} P_{J}(\infty)=1
$$

Substituting (10) in the last row of (9) to compute the steady-state probabilities, the expression for steadystate availability is given by

$$
\begin{aligned}
& A_{v 1}(\infty)=\frac{N_{3}}{D_{3}} \\
& N_{3}=\alpha_{1}^{2} \alpha_{2} \alpha_{3}\left(\alpha_{2}^{2} \alpha_{3}+\alpha_{2}^{2} \beta_{1}+\alpha_{2} \alpha_{3}^{2}+\alpha_{2} \alpha_{3} \beta_{2}+\alpha_{2} \alpha_{3} \beta_{1}+\alpha_{2} \alpha_{3} \beta_{3}+\alpha_{2} \beta_{1} \beta_{3}+\alpha_{3}^{2} \beta_{2}+\alpha_{3} \beta_{1} \beta_{2}+\alpha_{3} \beta_{2}^{2}\right) \\
& +\alpha_{1} \alpha_{2} \alpha_{3} \beta_{1}\left(\alpha_{2}^{2} \alpha_{3}+\alpha_{2}^{2} \beta_{1}+\alpha_{2} \alpha_{3}^{2}+\alpha_{2} \alpha_{3} \beta_{2}+\alpha_{2} \alpha_{3} \beta_{1}+\alpha_{2} \alpha_{3} \beta_{3}+\alpha_{2} \beta_{1} \beta_{3}+\alpha_{3}^{2} \beta_{2}+\alpha_{3} \beta_{1} \beta_{2}+\alpha_{3} \beta_{2}^{3}\right) \\
& +\alpha_{1} \alpha_{2} \alpha_{3} \beta_{1}\left(\alpha_{2}^{2} \beta_{3}+\alpha_{2} \alpha_{3} \beta_{3}+\alpha_{2} \beta_{2} \beta_{3}+\alpha_{2} \beta_{3}^{2}-\alpha_{2} \beta_{2}^{2}+\alpha_{3} \beta_{2} \beta_{3}\right) \\
& D_{3}=2 \alpha_{2}^{2} \alpha_{3} \beta_{1}^{2} \beta_{2} \beta_{3}+2 \alpha_{2} \alpha_{3}^{2} \beta_{1}^{2} \beta_{2} \beta_{3}+\alpha_{2} \alpha_{3} \beta_{1}^{3} \beta_{2} \beta_{3}+2 \alpha_{2} \alpha_{3} \beta_{1}^{2} \beta_{2}^{2} \beta_{3}+\alpha_{1}^{2} \alpha_{2}^{2} \alpha_{3}^{3}+3 \alpha_{2}^{2} \alpha_{3} \beta_{1}^{2} \beta_{3}^{2} \\
& +2 \alpha_{2}^{2} \alpha_{3} \beta_{1}^{2} \beta_{3}+\alpha_{1}^{2} \alpha_{2}^{3} \alpha_{3}^{2}+\alpha_{2} \alpha_{3}^{2} \beta_{1} \beta_{2}^{3}+\alpha_{2}^{2} \alpha_{3}^{2} \beta_{1}^{2}+2 \alpha_{2} \alpha_{3}^{2} \beta_{1} \beta_{2}^{2} \beta_{3}+\alpha_{2} \alpha_{3} \beta_{1} \beta_{2}^{3} \beta_{3}+\alpha_{2}^{2} \alpha_{3}^{2} \beta_{1} \beta_{2}^{2} \\
& +\alpha_{2}^{3} \beta_{1}^{3} \beta_{3}+\alpha_{2}^{3} \alpha_{3} \beta_{1}^{3}+\alpha_{2}^{3} \beta_{1}^{2} \beta_{3}^{2}+\alpha_{2}^{3} \alpha_{3}^{2} \beta_{1}^{2}+\alpha_{2}^{2} \beta_{1}^{3} \beta_{3}^{2}+\alpha_{2}^{2} \alpha_{3}^{2} \beta_{1}^{3}+\alpha_{2}^{2} \beta_{1}^{2} \beta_{3}^{2}+\alpha_{2}^{2} \beta_{3}^{2} \beta_{2} \beta_{3}^{2} \\
& +\alpha_{2}^{2} \alpha_{3}^{2} \beta_{1}^{2} \beta_{2}+\alpha_{2} \alpha_{3}^{2} \beta_{1}^{3} \beta_{2}+\alpha_{2} \alpha_{3}^{3} \beta_{1}^{2} \beta_{2}+\alpha_{2}^{2} \alpha_{3} \beta_{1} \beta_{2}^{2} \beta_{3}+\alpha_{2} \alpha_{3} \beta_{1} \beta_{2}^{2} \beta_{3}^{2}+\alpha_{1}^{2} \alpha_{3}^{2} \beta_{2}^{3}+\alpha_{1}^{2} \alpha_{3}^{3} \beta_{2}^{2} \\
& +\alpha_{1} \alpha_{2}^{3} \beta_{1}^{2} \beta_{3}+\alpha_{1} \alpha_{2}^{3} \alpha_{3} \beta_{1}^{2}+\alpha_{1} \alpha_{2}^{3} \alpha_{3}^{2} \beta_{1}+\alpha_{1} \alpha_{2}^{2} \beta_{1}^{2} \beta_{3}^{2}+\alpha_{1} \alpha_{2}^{2} \alpha_{3}^{2} \beta_{1}^{2}+\alpha_{1} \alpha_{2}^{2} \alpha_{3}^{3} \beta_{1}+\alpha_{1}^{2} \alpha_{2}^{3} \alpha_{3} \beta_{1} \\
& +\alpha_{1}^{2} \alpha_{2}^{2} \alpha_{3}^{2} \beta_{1}+\alpha_{1}^{2} \alpha_{2}^{2} \alpha_{3}^{2} \beta_{3}+2 \alpha_{1}^{2} \alpha_{2}^{2} \alpha_{3}^{2} \beta_{2}+\alpha_{2} \alpha_{3}^{3} \beta_{1} \beta_{2}^{2}+2 \alpha_{1}^{2} \alpha_{2} \alpha_{3}^{3} \beta_{2}+2 \alpha_{1}^{2} \alpha_{2} \alpha_{3}^{2} \beta_{2}^{2}+\alpha_{1}^{2} \alpha_{3}^{2} \beta_{1} \beta_{2}^{2} \\
& +\alpha_{1} \alpha_{2}^{2} \alpha_{3} \beta_{1} \beta_{3}+\alpha_{1} \alpha_{2}^{2} \beta_{1}^{2} \beta_{2} \beta_{3}+2 \alpha_{1} \alpha_{2}^{2} \alpha_{3} \beta_{1}^{2} \beta_{3}+\alpha_{1} \alpha_{2}^{2} \alpha_{3} \beta_{1}^{2} \beta_{2}+\alpha_{1} \alpha_{2}^{2} \alpha_{3} \beta_{1} \beta_{3}^{2}+2 \alpha_{1} \alpha_{2}^{2} \alpha_{3}^{2} \beta_{1} \beta_{3} \\
& +2 \alpha_{1} \alpha_{2}^{2} \alpha_{3} \beta \beta_{2} \beta_{3}+2 \alpha_{1} \alpha_{2}^{2} \alpha_{3}^{2} \beta_{1} \beta_{2}+2 \alpha_{1} \alpha_{2} \alpha_{3} \beta_{1}^{2} \beta_{2} \beta_{3}+2 \alpha_{2} \alpha_{3}^{2} \beta_{1}^{2} \beta_{2}^{2}+2 \alpha_{1} \alpha_{2} \alpha_{3}^{2} \beta_{1}^{2} \beta_{2}+\alpha_{1} \alpha_{2} \alpha_{3} \beta_{1} \beta_{2} \beta_{3}^{2} \\
& +3 \alpha_{1} \alpha_{2} \alpha_{3}^{2} \beta_{1} \beta_{2} \beta_{3}+2 \alpha_{1} \alpha_{2} \alpha_{3} \beta_{1} \beta_{2}^{2} \beta_{3}+2 \alpha_{1} \alpha_{2} \alpha_{3}^{3} \beta_{1} \beta_{2}+2 \alpha_{1} \alpha_{2} \alpha_{3}^{2} \beta_{1} \beta_{2}^{2}+\alpha_{1}^{2} \alpha_{2}^{2} \alpha_{3} \beta_{1} \beta_{3}+\alpha_{1}^{2} \alpha_{2}^{2} \alpha_{3} \beta_{1} \beta_{2} \\
& +\alpha_{1}^{2} \alpha_{2} \alpha_{3} \beta_{1} \beta_{2} \beta_{3}+2 \alpha_{1}^{2} \alpha_{2} \alpha_{3}^{2} \beta_{1} \beta_{2}+\alpha_{1}^{2} \alpha_{2} \alpha_{3}^{2} \beta_{2} \beta_{3}+\alpha_{2} \alpha_{3} \beta_{1}^{2} \beta_{2} \beta_{3}^{2}+3 \alpha_{2}^{2} \alpha_{3}^{2} \beta_{1}^{2} \beta_{3}+2 \alpha_{2}^{2} \alpha_{3} \beta_{1}^{3} \beta_{3}
\end{aligned}
$$

\subsection{Availability Model Formulation for Configuration II}

For the analysis of availability case of configuration II we use the same initial condition as in Subsection 3.2

$$
P(0)=\left[P_{0}(0), P_{1}(0), P_{2}(0), P_{3}(0), P_{4}(0), \cdots, P_{10}(0)\right]=[1,0,0,0,0,0,0,0,0,0,0]
$$

The differential equations are expressed in the form

$$
\left[\begin{array}{c}
\dot{P}_{0} \\
\dot{P}_{1} \\
\dot{P}_{2} \\
\dot{P}_{3} \\
\dot{P}_{4} \\
\dot{P}_{5} \\
\dot{P}_{6} \\
\dot{P}_{7} \\
\dot{P}_{8} \\
\dot{P}_{9} \\
\dot{P}_{10}
\end{array}\right]=\left[\begin{array}{ccccccccccc}
-\left(\beta_{1}+\beta_{2}\right) & \alpha_{1} & \alpha_{2} & 0 & 0 & 0 & 0 & 0 & 0 & 0 & 0 \\
\beta_{1} & -y_{1} & 0 & \alpha_{3} & \alpha_{1} & \alpha_{2} & 0 & 0 & 0 & 0 & 0 \\
\beta_{2} & 0 & -\alpha_{2} & 0 & 0 & 0 & 0 & 0 & 0 & 0 & 0 \\
0 & \beta_{3} & 0 & -y_{2} & 0 & 0 & \alpha_{2} & \alpha_{1} & \alpha_{3} & 0 & 0 \\
0 & \beta_{1} & 0 & 0 & -y_{3} & 0 & 0 & \alpha_{3} & 0 & 0 & 0 \\
0 & \beta_{2} & 0 & 0 & 0 & -\alpha_{2} & 0 & 0 & 0 & 0 & 0 \\
0 & 0 & 0 & \beta_{2} & 0 & 0 & -\alpha_{2} & 0 & 0 & 0 & 0 \\
0 & 0 & 0 & \beta_{1} & \beta_{3} & 0 & 0 & -y_{4} & 0 & \alpha_{3} & 0 \\
0 & 0 & 0 & \beta_{3} & 0 & 0 & 0 & 0 & -y_{5} & 0 & \alpha_{2} \\
0 & 0 & 0 & 0 & 0 & 0 & 0 & \beta_{3} & 0 & -\alpha_{3} & 0 \\
0 & 0 & 0 & 0 & 0 & 0 & 0 & 0 & \beta_{2} & 0 & -\alpha_{2}
\end{array}\right]\left[\begin{array}{c}
P_{0} \\
P_{1} \\
P_{2} \\
P_{3} \\
P_{4} \\
P_{5} \\
P_{6} \\
P_{7} \\
P_{8} \\
P_{9} \\
P_{10}
\end{array}\right]
$$

The steady-state availability is given by

$$
A_{V 2}(\infty)=P_{0}(\infty)+P_{1}(\infty)+P_{3}(\infty)+P_{8}(\infty)
$$

In the steady-state, the derivatives of the state probabilities become zero and therefore Equation (4) become 


$$
T_{2} P=0
$$

which is in matrix form

$$
\left[\begin{array}{ccccccccccc}
-\left(\beta_{1}+\beta_{2}\right) & \alpha_{1} & \alpha_{2} & 0 & 0 & 0 & 0 & 0 & 0 & 0 & 0 \\
\beta_{1} & -y_{1} & 0 & \alpha_{3} & \alpha_{1} & \alpha_{2} & 0 & 0 & 0 & 0 & 0 \\
\beta_{2} & 0 & -\alpha_{2} & 0 & 0 & 0 & 0 & 0 & 0 & 0 & 0 \\
0 & \beta_{3} & 0 & -y_{2} & 0 & 0 & \alpha_{2} & \alpha_{1} & \alpha_{3} & 0 & 0 \\
0 & \beta_{1} & 0 & 0 & -y_{3} & 0 & 0 & \alpha_{3} & 0 & 0 & 0 \\
0 & \beta_{2} & 0 & 0 & 0 & -\alpha_{2} & 0 & 0 & 0 & 0 & 0 \\
0 & 0 & 0 & \beta_{2} & 0 & 0 & -\alpha_{2} & 0 & 0 & 0 & 0 \\
0 & 0 & 0 & \beta_{1} & \beta_{3} & 0 & 0 & -y_{4} & 0 & \alpha_{3} & 0 \\
0 & 0 & 0 & \beta_{3} & 0 & 0 & 0 & 0 & -y_{5} & 0 & \alpha_{2} \\
0 & 0 & 0 & 0 & 0 & 0 & 0 & \beta_{3} & 0 & -\alpha_{3} & 0 \\
0 & 0 & 0 & 0 & 0 & 0 & 0 & 0 & \beta_{2} & 0 & -\alpha_{2}
\end{array}\right]\left[\begin{array}{c}
P_{0}(\infty) \\
P_{1}(\infty) \\
P_{2}(\infty) \\
P_{3}(\infty) \\
P_{4}(\infty) \\
P_{5}(\infty) \\
P_{6}(\infty) \\
P_{7}(\infty) \\
P_{8}(\infty) \\
P_{9}(\infty) \\
P_{10}(\infty)
\end{array}\right]=\left[\begin{array}{l}
0 \\
0 \\
0 \\
0 \\
0 \\
0 \\
0 \\
0 \\
0 \\
0 \\
0
\end{array}\right]
$$

Using the following normalizing condition

$$
\sum_{J=0}^{10} P_{J}(\infty)=1
$$

Substituting (14) in the last row of (13) to compute the steady-state probabilities, the expression for steadystate availability is given by

$$
\begin{aligned}
& A_{V 2}(\infty)=\frac{N_{4}}{D_{4}} \\
& N_{4}=\alpha_{1}^{2} \alpha_{2} \alpha_{3}^{2}\left(\alpha_{1} \alpha_{3}+\alpha_{1} \beta_{1}+\alpha_{3}^{2}+\alpha_{3} \beta_{3}+2 \alpha_{3} \beta_{1}+\beta_{1}^{2}+2 \beta_{1} \beta_{3}\right) \\
& +\alpha_{1} \alpha_{2} \alpha_{3}^{2} \beta_{1}\left(\alpha_{1} \alpha_{3}+\alpha_{1} \beta_{1}+\alpha_{3}^{2}+\alpha_{3} \beta_{3}+2 \alpha_{3} \beta_{1}+\beta_{1}^{2}+2 \beta_{1} \beta_{3}\right) \\
& +\alpha_{1} \alpha_{2} \alpha_{3} \beta_{1} \beta_{3}\left(\alpha_{1} \alpha_{3}+\alpha_{1} \beta_{1}+\alpha_{3}^{2}+2 \alpha_{3} \beta_{1}+\alpha_{3} \beta_{3}+\beta_{1}^{2}+\beta_{1} \beta_{3}\right) \\
& +\alpha_{1} \alpha_{2} \alpha_{3} \beta_{1} \beta_{3}^{2}\left(\alpha_{1}+\alpha_{3}+\beta_{1}+\beta_{3}\right) \\
& D_{4}=\alpha_{1}^{3} \alpha_{3}^{3} \beta_{2}+\alpha_{1}^{2} \alpha_{2} \beta_{1}^{2} \beta_{3}^{2}+2 \alpha_{1}^{2} \alpha_{2} \alpha_{3}^{2} \beta_{1}^{2}+3 \alpha_{1}^{2} \alpha_{2} \alpha_{3}^{3} \beta_{1}+\alpha_{1}^{2} \alpha_{2} \alpha_{3}^{3} \beta_{3}+\alpha_{1}^{2} \alpha_{3}^{4} \beta_{2}+2 \alpha_{1} \alpha_{2} \beta_{1}^{3} \beta_{3}^{2} \\
& +\alpha_{2} \beta_{1}^{4} \beta_{3}^{2}+\alpha_{2} \alpha_{3}^{2} \beta_{1}^{4}+2 \alpha_{2} \beta_{1}^{3} \beta_{3}^{3}+2 \alpha_{2} \alpha_{3}^{3} \beta_{1}^{3}+\alpha_{2} \beta_{1}^{2} \beta_{3}^{4}+\alpha_{2} \alpha_{3}^{4} \beta_{1}^{2}+\alpha_{1}^{2} \alpha_{2} \alpha_{3}^{4}+2 \alpha_{1} \alpha_{2} \alpha_{3}^{2} \beta_{1}^{3} \\
& +\alpha_{1}^{3} \alpha_{2} \alpha_{3}^{2} \beta_{1}+2 \alpha_{1} \alpha_{2} \alpha_{3}^{2} \beta_{1} \beta_{3}^{2}+2 \alpha_{1} \alpha_{2} \beta_{1}^{2} \beta_{3}^{3}+3 \alpha_{1} \alpha_{2} \alpha_{3}^{3} \beta_{1}^{2}+\alpha_{1} \alpha_{2} \alpha_{3}^{4} \beta_{1}+\alpha_{2} \alpha_{3} \beta_{1}^{4} \beta_{3}+4 \alpha_{2} \alpha_{3} \beta_{1}^{3} \beta_{3}^{2} \\
& +4 \alpha_{2} \alpha_{3}^{2} \beta_{1}^{3} \beta_{3}+2 \alpha_{2} \alpha_{3} \beta_{1}^{2} \beta_{3}^{3}+\alpha_{1}^{2} \alpha_{3}^{3} \beta_{2} \beta_{3}+3 \alpha_{2} \alpha_{3}^{2} \beta_{1}^{2} \beta_{3}^{2}+2 \alpha_{2} \alpha_{3}^{3} \beta_{1}^{2} \beta_{3}+2 \alpha_{1} \alpha_{2} \alpha_{3} \beta_{1}^{3} \beta_{3}+5 \alpha_{1} \alpha_{2} \alpha_{3} \beta_{1}^{2} \beta_{3}^{2} \\
& +5 \alpha_{1} \alpha_{2} \alpha_{3}^{2} \beta_{1}^{2} \beta_{3}+\alpha_{1} \alpha_{2} \alpha_{3} \beta_{1} \beta_{3}^{3}+2 \alpha_{1} \alpha_{2} \alpha_{3}^{3} \beta_{1} \beta_{3}+\alpha_{1} \alpha_{3} \beta_{1}^{3} \beta_{2} \beta_{3}+\alpha_{1} \alpha_{3}^{2} \beta_{1}^{3} \beta_{2}+2 \alpha_{1} \alpha_{3} \beta_{1}^{2} \beta_{2} \beta_{3}^{2} \\
& +4 \alpha_{1} \alpha_{3}^{2} \beta_{1}^{2} \beta_{2} \beta_{3}+2 \alpha_{1} \alpha_{3}^{3} \beta_{1}^{2} \beta_{2}+\alpha_{1}^{3} \alpha_{2} \alpha_{3}^{3}+\alpha_{1} \alpha_{3} \beta_{1} \beta_{2} \beta_{3}^{3}+\alpha_{1}^{3} \alpha_{3}^{2} \beta_{1} \beta_{2}+2 \alpha_{1} \alpha_{3}^{2} \beta_{1} \beta_{2} \beta_{3}^{2}+2 \alpha_{1} \alpha_{3}^{3} \beta_{1} \beta_{2} \beta_{3} \\
& +\alpha_{1} \alpha_{3}^{4} \beta_{1} \beta_{2}+\alpha_{1}^{2} \alpha_{2} \alpha_{3} \beta_{1}^{2} \beta_{3}+\alpha_{1}^{2} \alpha_{2} \alpha_{3} \beta_{1} \beta_{3}^{2}+3 \alpha_{1}^{2} \alpha_{2} \alpha_{3}^{2} \beta_{1} \beta_{3}+\alpha_{1}^{2} \alpha_{3} \beta_{1}^{2} \beta_{2} \beta_{3}+2 \alpha_{1}^{2} \alpha_{3}^{2} \beta_{1}^{2} \beta_{2} \\
& +\alpha_{1}^{2} \alpha_{3} \beta_{1} \beta_{2} \beta_{3}^{2}+3 \alpha_{1}^{2} \alpha_{3}^{3} \beta_{1} \beta_{2}+3 \alpha_{1}^{2} \alpha_{3}^{2} \beta_{1} \beta_{2} \beta_{3}
\end{aligned}
$$

\subsection{Availability Model Formulation for Configuration III}

For the analysis of availability case of configuration III we use the same initial condition as in Subsection 3.3

$$
P(0)=\left[P_{0}(0), P_{1}(0), P_{2}(0), P_{3}(0), P_{4}(0), P_{5}(0), P_{6}(0), P_{7}(0)\right]=[1,0,0,0,0,0,0,0]
$$

The differential equations are expressed in the form 


$$
\left[\begin{array}{c}
\dot{P}_{0} \\
\dot{P}_{1} \\
\dot{P}_{2} \\
\dot{P}_{3} \\
\dot{P}_{4} \\
\dot{P}_{5} \\
\dot{P}_{6} \\
\dot{P}_{7}
\end{array}\right]=\left[\begin{array}{cccccccc}
-\left(\beta_{1}+\beta_{2}\right) & \alpha_{1} & \alpha_{2} & 0 & 0 & 0 & 0 & 0 \\
\beta_{1} & -\left(\alpha_{1}+\beta_{1}+\beta_{2}+\beta_{3}\right) & 0 & \alpha_{1} & \alpha_{2} & \alpha_{3} & 0 & 0 \\
\beta_{2} & 0 & -\alpha_{2} & 0 & 0 & 0 & 0 & 0 \\
0 & \beta_{1} & 0 & -\left(\alpha_{1}+\beta_{3}\right) & 0 & 0 & \alpha_{3} & 0 \\
0 & \beta_{2} & 0 & 0 & -\left(\alpha_{2}+\beta_{3}\right) & 0 & 0 & \alpha_{3} \\
0 & \beta_{3} & 0 & 0 & 0 & -\left(\alpha_{3}+\beta_{1}+\beta_{2}\right) & 0 & \alpha_{2} \\
0 & 0 & 0 & \beta_{3} & 0 & \beta_{1} & -\alpha_{3} & 0 \\
0 & 0 & 0 & 0 & \beta_{3} & \beta_{2} & 0 & -\left(\alpha_{2}+\alpha_{3}\right)
\end{array}\right]\left[\begin{array}{c}
P_{0} \\
P_{1} \\
P_{2} \\
P_{3} \\
P_{4} \\
P_{5} \\
P_{6} \\
P_{7}
\end{array}\right]
$$

The steady-state availability is given by

$$
A_{v 3}(\infty)=P_{0}(\infty)+P_{1}(\infty)+P_{5}(\infty)
$$

In the steady-state, the derivatives of the state probabilities become zero and therefore Equation (6) become

$$
T_{3} P=0
$$

which is in matrix form

$$
\left[\begin{array}{cccccccc}
-\left(\beta_{1}+\beta_{2}\right) & \alpha_{1} & \alpha_{2} & 0 & 0 & 0 & 0 & 0 \\
\beta_{1} & -\left(\alpha_{1}+\beta_{1}+\beta_{2}+\beta_{3}\right) & 0 & \alpha_{1} & \alpha_{2} & \alpha_{3} & 0 & 0 \\
\beta_{2} & 0 & -\alpha_{2} & 0 & 0 & 0 & 0 & 0 \\
0 & \beta_{1} & 0 & -\left(\alpha_{1}+\beta_{3}\right) & 0 & 0 & \alpha_{3} & 0 \\
0 & \beta_{2} & 0 & 0 & -\left(\alpha_{2}+\beta_{3}\right) & 0 & 0 & \alpha_{3} \\
0 & \beta_{3} & 0 & 0 & 0 & -\left(\alpha_{3}+\beta_{1}+\beta_{2}\right) & 0 & \alpha_{2} \\
0 & 0 & 0 & \beta_{3} & 0 & \beta_{1} & -\alpha_{3} & 0 \\
0 & 0 & 0 & 0 & \beta_{3} & \beta_{2} & 0 & -\left(\alpha_{2}+\alpha_{3}\right)
\end{array}\right]\left[\begin{array}{l}
P_{0}(\infty) \\
P_{1}(\infty) \\
P_{2}(\infty) \\
P_{3}(\infty) \\
P_{4}(\infty) \\
P_{5}(\infty) \\
P_{6}(\infty) \\
P_{7}(\infty)
\end{array}\right]=\left[\begin{array}{l}
0 \\
0 \\
0 \\
0 \\
0 \\
0 \\
0 \\
0
\end{array}\right]
$$

Using the following normalizing condition

$$
\sum_{J=0}^{7} P_{J}(\infty)=1
$$

Substituting (18) in the last row of (17) to compute the steady-state probabilities, the expression for steadystate availability is given by

$$
\begin{aligned}
& A_{v 3}(\infty)=\frac{N_{5}}{D_{5}} \\
& N_{5}=\alpha_{1}^{2} \alpha_{2} \alpha_{3}\left(\alpha_{2} \beta_{1}+\alpha_{2} \alpha_{3}+\alpha_{3}^{2}+\alpha_{3} \beta_{1}+\alpha_{3} \beta_{3}+\alpha_{3} \beta_{2}+\beta_{1} \beta_{3}\right)+\alpha_{1} \alpha_{2} \alpha_{3} \beta_{1} \beta_{3}\left(\alpha_{2}+\alpha_{3}+\beta_{1}+\beta_{3}\right) \\
& +\alpha_{1} \alpha_{2} \alpha_{3} \beta_{1}\left(\alpha_{2} \beta_{1}+\alpha_{2} \alpha_{3}+\alpha_{3}^{2}+\alpha_{3} \beta_{1}+\alpha_{3} \beta_{3}+\alpha_{3} \beta_{2}+\beta_{1} \beta_{3}\right) \\
& D_{5}=\alpha_{2} \beta_{1}^{3} \beta_{3}^{2}+\alpha_{2} \beta_{1}^{2} \beta_{3}^{3}+\alpha_{1}^{2} \alpha_{2} \alpha_{3}^{2} \beta_{1}+\alpha_{1}^{2} \alpha_{2} \alpha_{3}^{3}+2 \alpha_{1} \alpha_{2} \alpha_{3}^{2} \beta_{1} \beta_{3}+\alpha_{1} \alpha_{2} \beta_{1}^{2} \beta_{3}^{2}+\alpha_{1}^{2} \alpha_{3}^{2} \beta_{2}^{2}+\alpha_{2} \alpha_{3}^{2} \beta_{1}^{2} \beta_{2} \\
& +\alpha_{1} \alpha_{3} \beta_{1} \beta_{2}^{2} \beta_{3}+\alpha_{2}^{2} \alpha_{3} \beta_{1}^{3}+\alpha_{1} \alpha_{2}^{2} \alpha_{3} \beta_{1} \beta_{3}+2 \alpha_{1} \alpha_{2} \alpha_{3} \beta_{1} \beta_{2} \beta_{3}+2 \alpha_{1} \alpha_{2} \alpha_{3}^{2} \beta_{1} \beta_{2}+\alpha_{1}^{2} \alpha_{2} \alpha_{3} \beta_{1} \beta_{2}+\alpha_{1} \alpha_{2} \alpha_{3}^{3} \beta_{1} \\
& +\alpha_{1} \alpha_{2} \alpha_{3}^{2} \beta_{1}^{2}+\alpha_{1} \alpha_{2} \beta_{1}^{2} \beta_{2} \beta_{3}+\alpha_{1}^{2} \alpha_{3}^{2} \beta_{1} \beta_{2}+\alpha_{1} \alpha_{3}^{2} \beta_{1} \beta_{2}^{2}+\alpha_{2} \alpha_{3}^{2} \beta_{1}^{3}+\alpha_{2} \alpha_{3}^{3} \beta_{1}^{2}+\alpha_{1} \alpha_{3}^{2} \beta_{1}^{2} \beta_{2}+\alpha_{1} \alpha_{3}^{3} \beta_{1} \beta_{2} \\
& +\alpha_{1}^{2} \alpha_{3} \beta_{1} \beta_{2} \beta_{3}+2 \alpha_{1} \alpha_{3}^{2} \beta_{1} \beta_{2} \beta_{3}+3 \alpha_{2} \alpha_{3}^{2} \beta_{1}^{2} \beta_{3}+2 \alpha_{2} \alpha_{3} \beta_{1}^{3} \beta_{3}+3 \alpha_{2} \alpha_{3} \beta_{1}^{2} \beta_{3}^{2}+\alpha_{2}^{2} \beta_{1}^{3} \beta_{3}+2 \alpha_{2}^{2} \alpha_{3} \beta_{1}^{2} \beta_{3} \\
& +\alpha_{1} \alpha_{2}^{2} \beta_{1}^{2} \beta_{3}+\alpha_{1} \alpha_{2}^{2} \alpha_{3} \beta_{1}^{2}+\alpha_{1}^{2} \alpha_{2}^{2} \alpha_{3} \beta_{1}+\alpha_{1} \alpha_{2}^{2} \alpha_{3}^{2} \beta_{1}+2 \alpha_{1}^{2} \alpha_{2} \alpha_{3}^{2} \beta_{2}+\alpha_{2}^{2} \beta_{1}^{2} \beta_{3}^{2}+\alpha_{2}^{2} \alpha_{3}^{2} \beta_{1}^{2}+\alpha_{1}^{2} \alpha_{2}^{2} \alpha_{3}^{2} \\
& +\alpha_{1}^{2} \alpha_{2} \alpha_{3}^{2} \beta_{3}+\alpha_{1}^{2} \alpha_{3}^{3} \beta_{2}+2 \alpha_{2} \alpha_{3} \beta_{1}^{2} \beta_{2} \beta_{3}+\alpha_{1} \alpha_{2} \alpha_{3} \beta_{1}^{2} \beta_{2}+\alpha_{2} \beta_{1}^{2} \beta_{2} \beta_{3}^{2}+2 \alpha_{1} \alpha_{2} \alpha_{3} \beta_{1}^{2} \beta_{3}+\alpha_{1} \alpha_{2} \alpha_{3} \beta_{1} \beta_{3}^{2} \\
& +\alpha_{1}^{2} \alpha_{2} \alpha_{3} \beta_{1} \beta_{3}+\alpha_{1} \alpha_{3} \beta_{1}^{2} \beta_{2} \beta_{3}+\alpha_{1}^{2} \alpha_{3}^{2} \beta_{2} \beta_{3}+\alpha_{1} \alpha_{3} \beta_{1} \beta_{2} \beta_{3}^{2}
\end{aligned}
$$

\section{Comparison of the Three Configurations}

In this section, we numerically compare the results for availability and MTSF for the developed models for the 
three configurations.

Case I:

We fix $\beta_{1}=0.2, \beta_{2}=0.4, \beta_{3}=0.4, \alpha_{2}=0.7, \alpha_{3}=0.9$ and vary $\alpha_{1}$ between 0 to 1 for Figure 1 , $\beta_{2}=0.4, \beta_{3}=0.4, \alpha_{1}=0.5, \alpha_{2}=0.7, \alpha_{3}=0.9$ and vary $\beta_{1}$ between 0 to 1 for Figure 2 .

Case II:

We fix $\beta_{1}=0.02, \beta_{2}=0.04, \beta_{3}=0.04, \alpha_{1}=0.05, \alpha_{3}=0.09$ and vary $\alpha_{2}$ between 0 to 1 for Figure 3 and $\beta_{1}=0.02, \beta_{3}=0.04, \alpha_{1}=0.05, \alpha_{3}=0.3, \alpha_{3}=0.09$ and vary $\beta_{2}$ between 0 to 1 for Figure 4 .

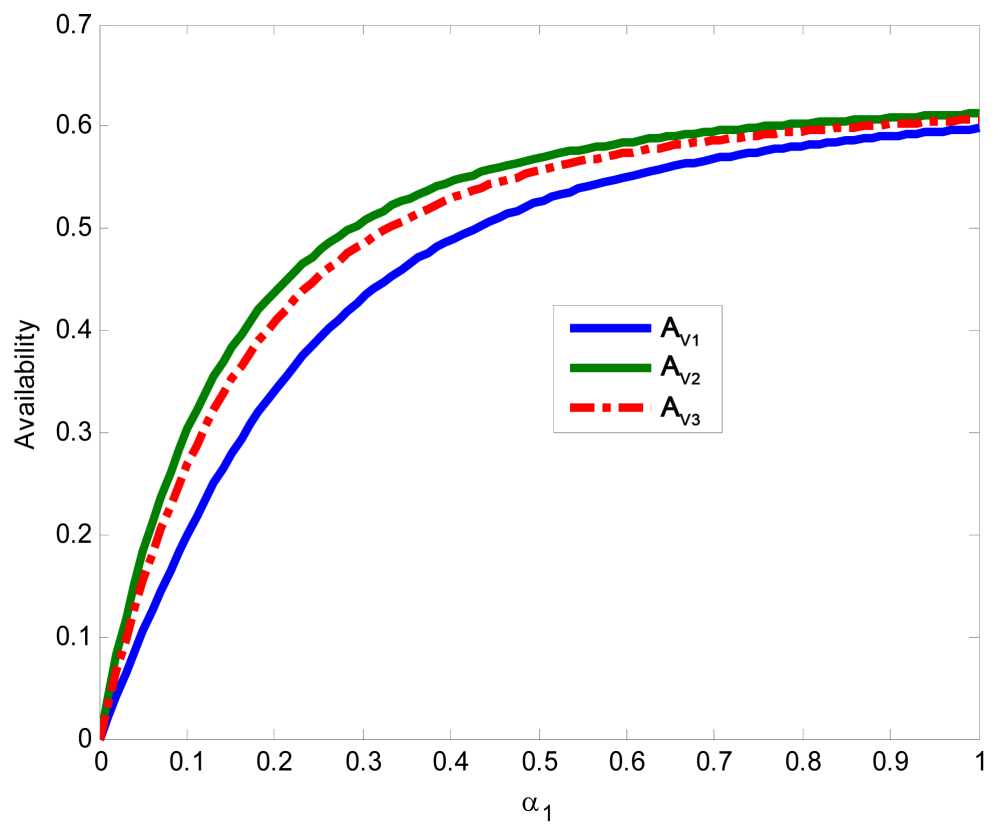

Figure 1. Availability against $\alpha_{1}$.

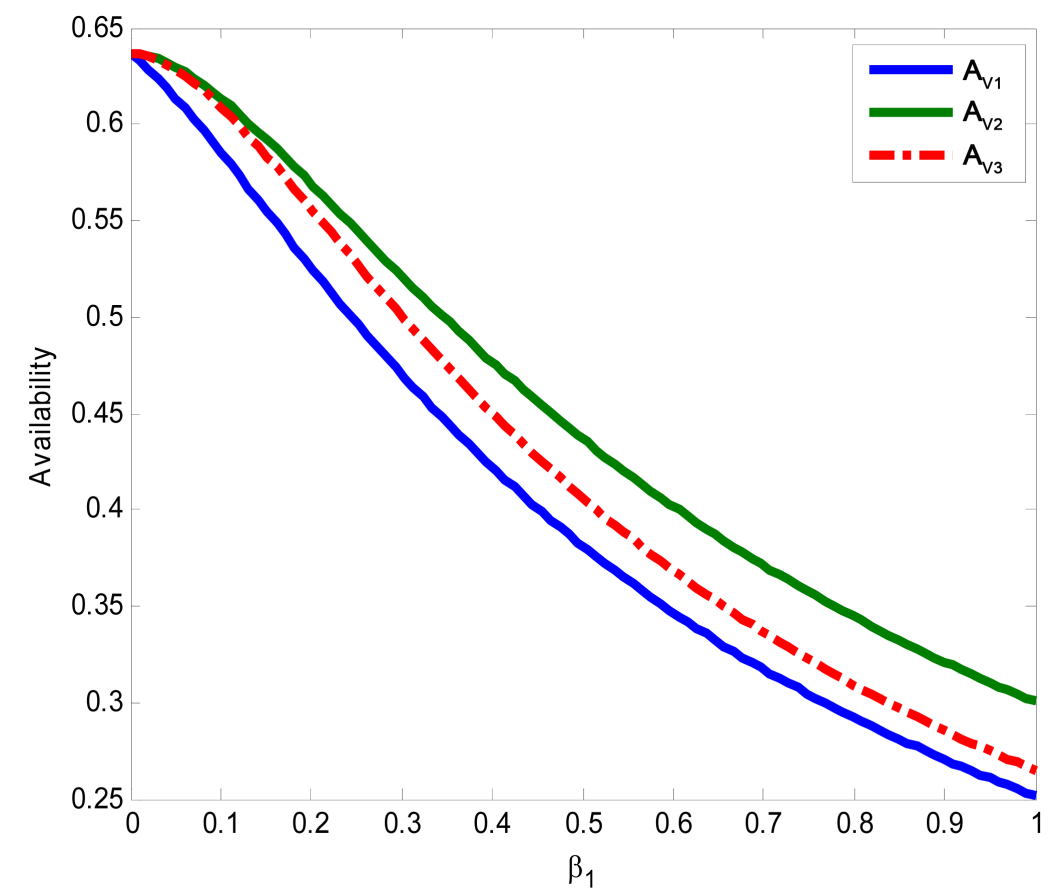

Figure 2. Availability against $\beta_{1}$. 


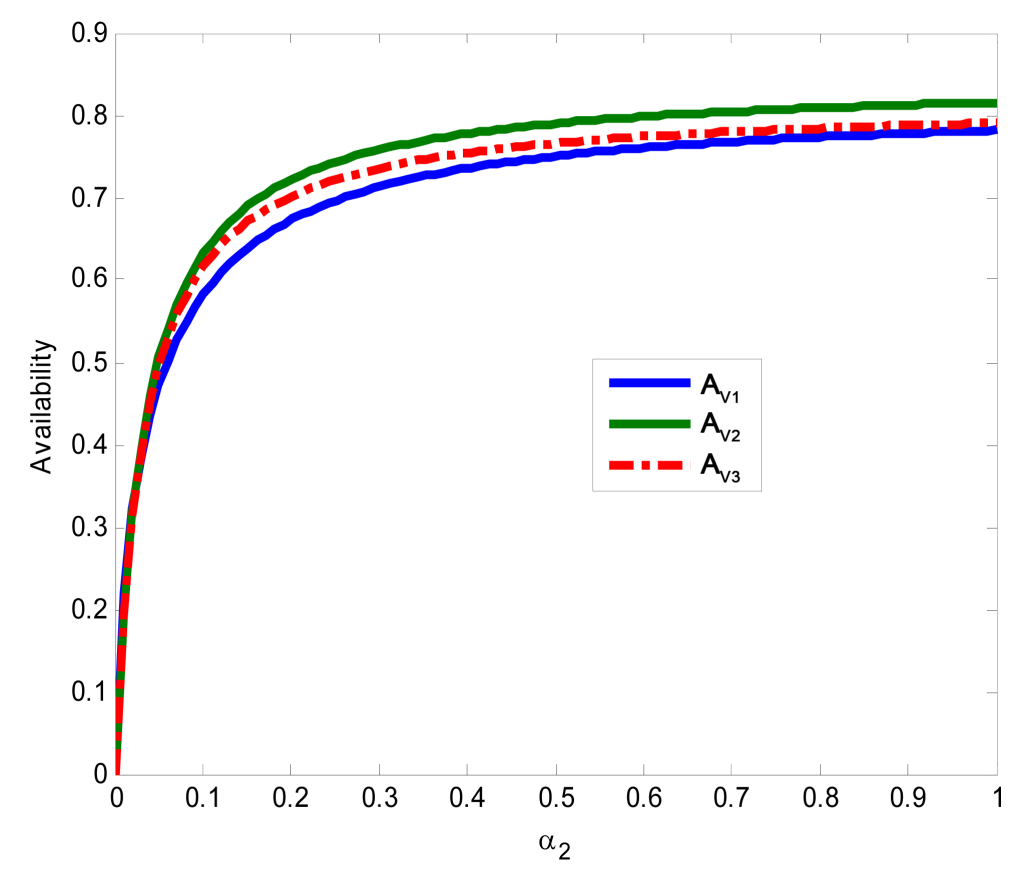

Figure 3. Availability against $\alpha_{2}$.

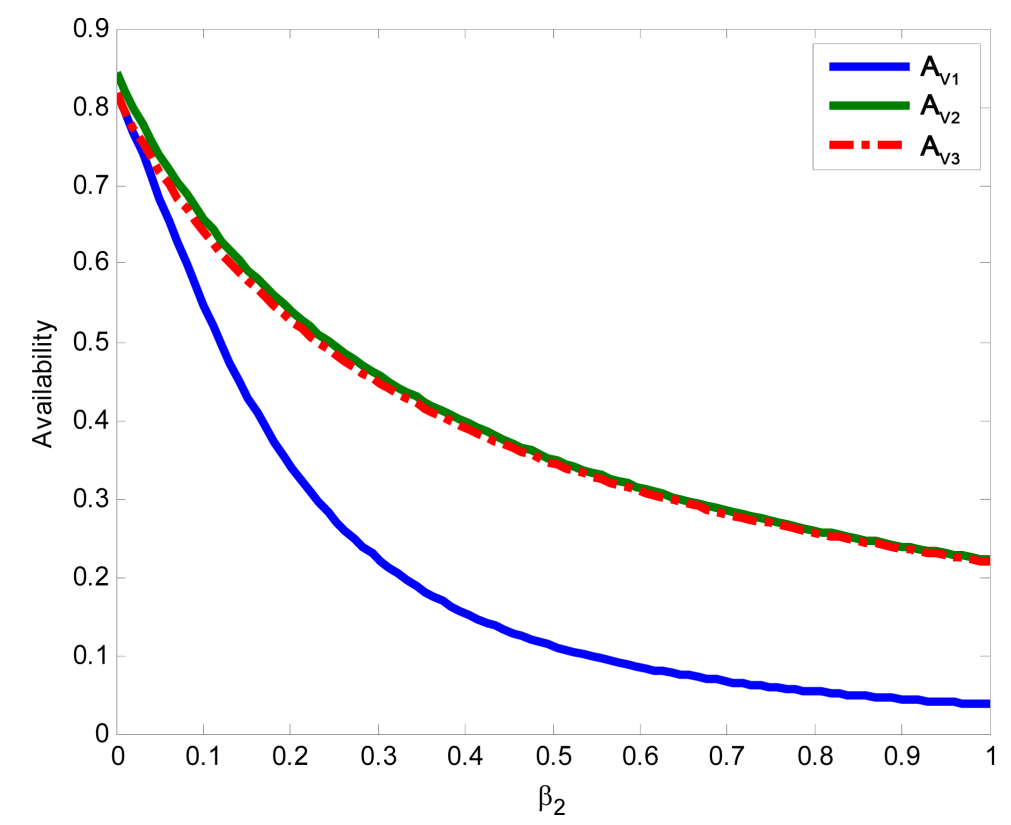

Figure 4. Availability against $\beta_{2}$.

\section{Case III:}

We fix $\beta_{1}=0.2, \beta_{2}=0.4, \beta_{3}=0.4, \alpha_{1}=0.2, \alpha_{2}=0.3$ and vary $\alpha_{3}$ between 0 to 1 for Figure 5 and $\beta_{1}=0.2, \beta_{2}=0.4, \alpha_{1}=0.2, \alpha_{2}=0.3, \alpha_{3}=0.4$ and vary $\beta_{3}$ between 0 to 1 for Figure 6 .

Case IV:

We fix $\beta_{1}=0.02, \beta_{2}=0.04, \beta_{3}=0.9, \alpha_{3}=0.8$ and vary $\alpha_{1}$ between 0 to 1 for Figure $7, \beta_{1}=0.02$, $\beta_{2}=0.04, \beta_{3}=0.9, \alpha_{1}=0.9$ and vary $\alpha_{3}$ between 0 to 1 for Figure 8 and $\beta_{1}=0.02, \beta_{2}=0.04$, $\alpha_{1}=0.9, \alpha_{3}=0.8$ and vary $\beta_{3}$ between 0 to 1 for Figure 9, and vary $\beta_{1}$ and $\beta_{2}$ for Figures 9-11.

From Figure 1, the availability results for the three systems being studied against the repair rate $\alpha_{1}$. It is 


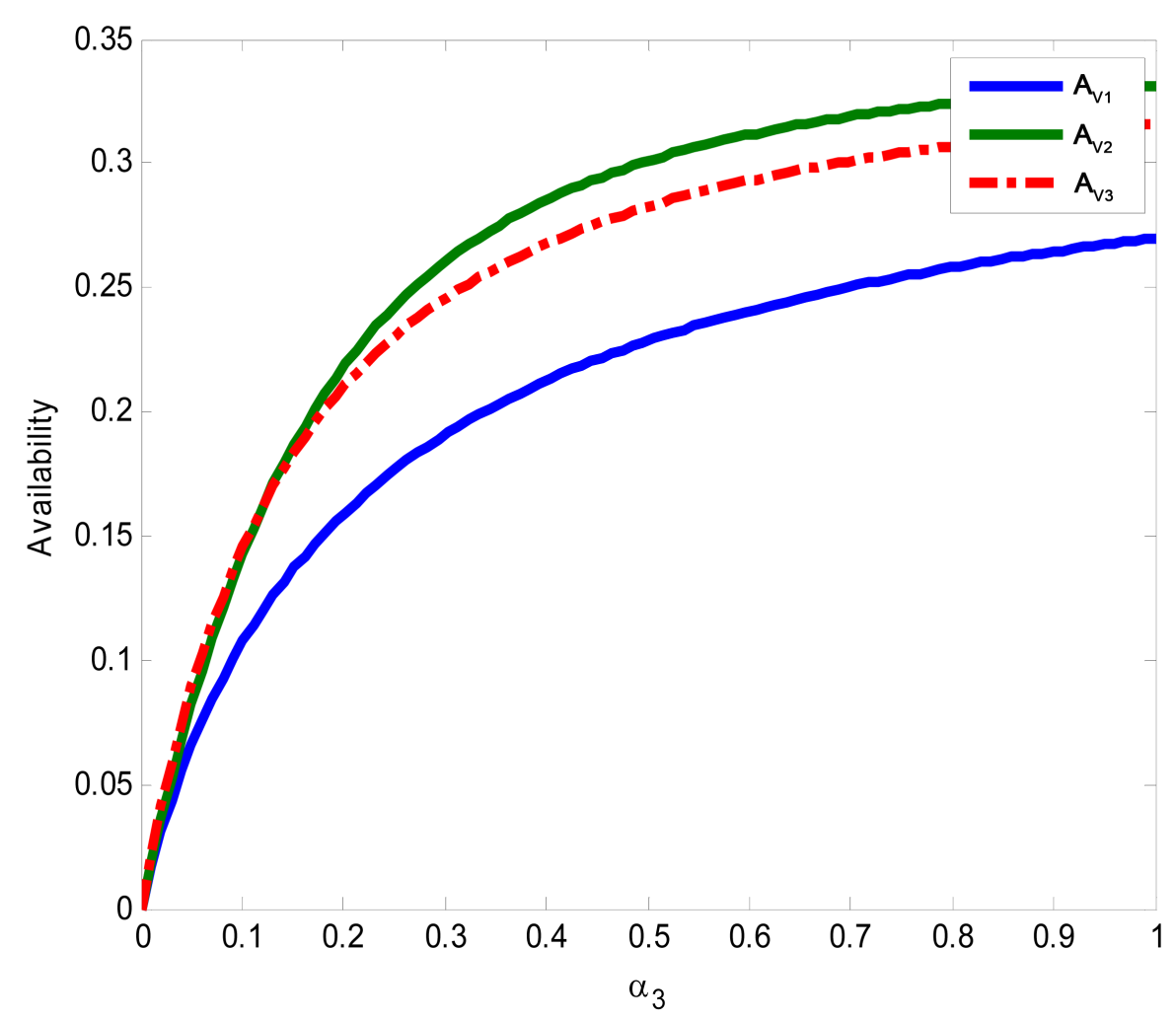

Figure 5. Availability against $\alpha_{3}$.

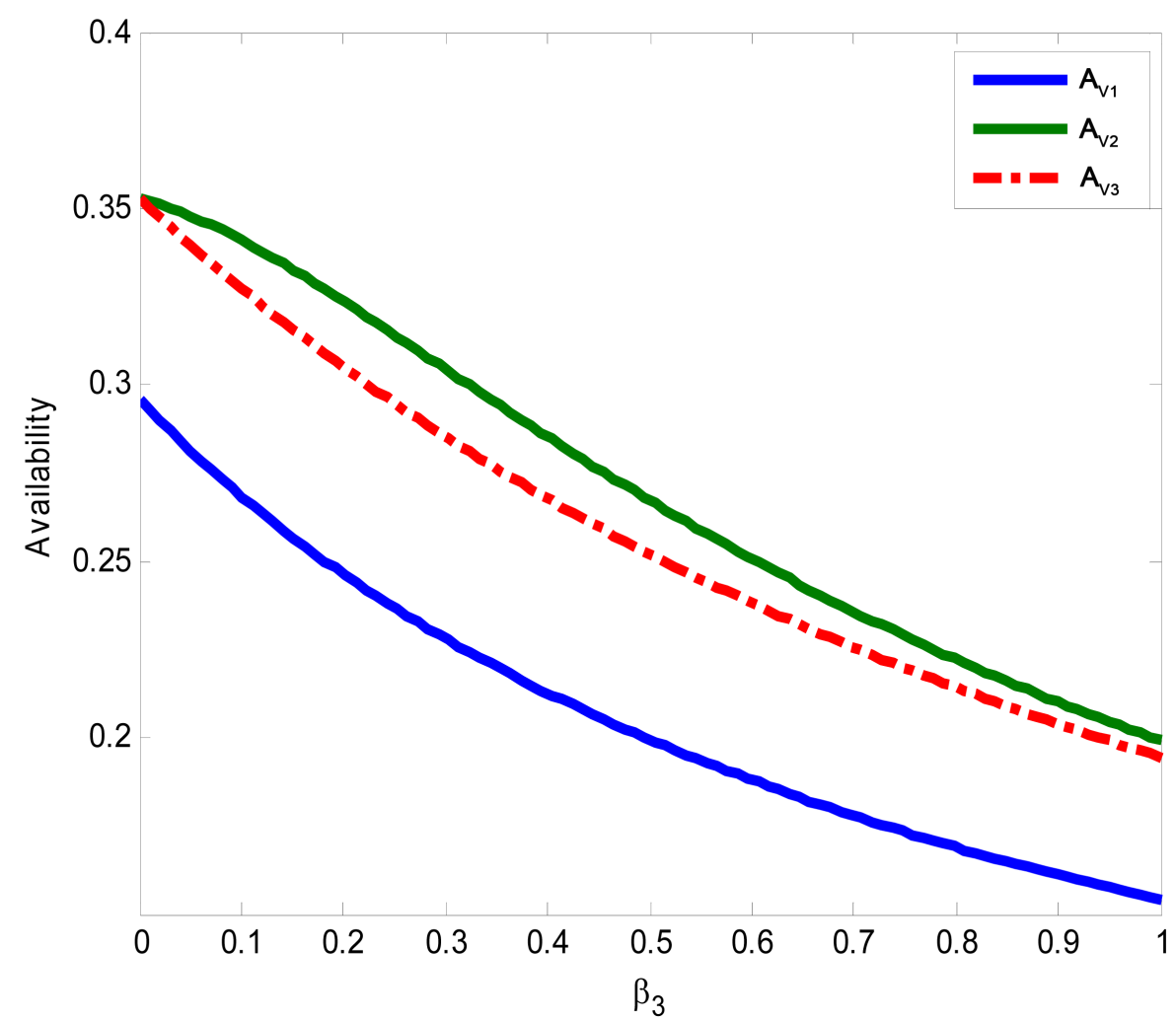

Figure 6. Availability against $\beta_{3}$. 


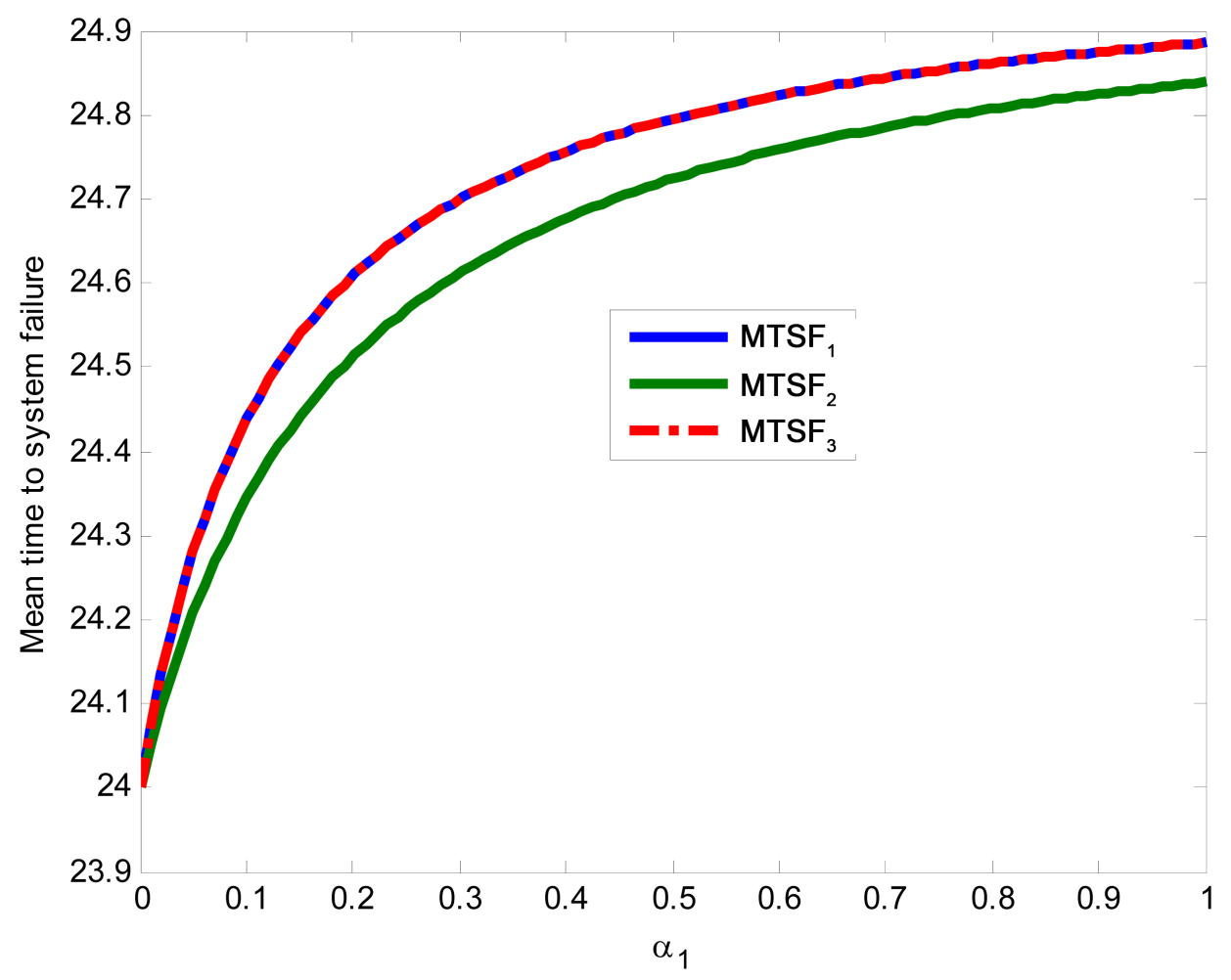

Figure 7. MTSF against $\alpha_{1}$.

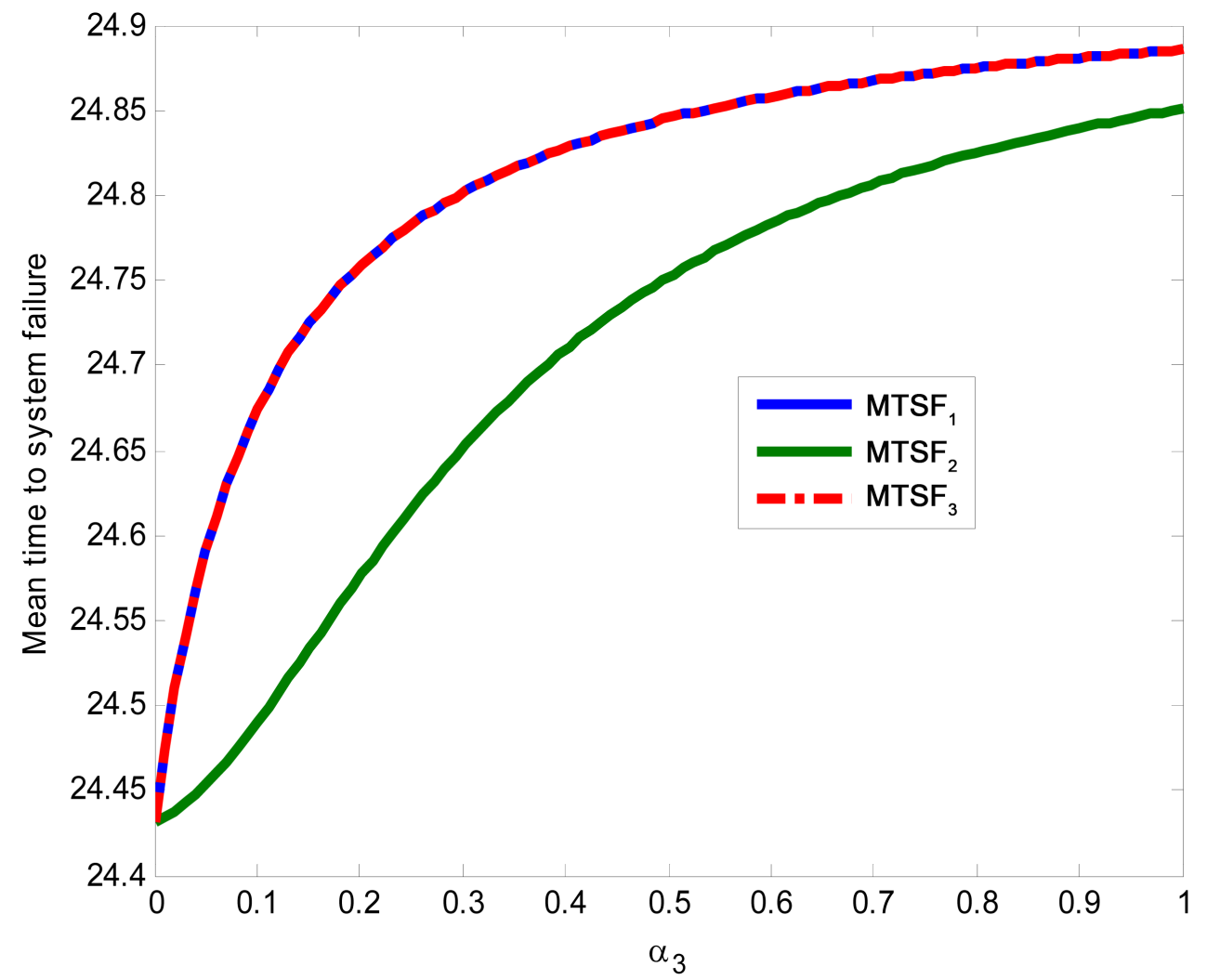

Figure 8 . MTSF against $\alpha_{3}$. 


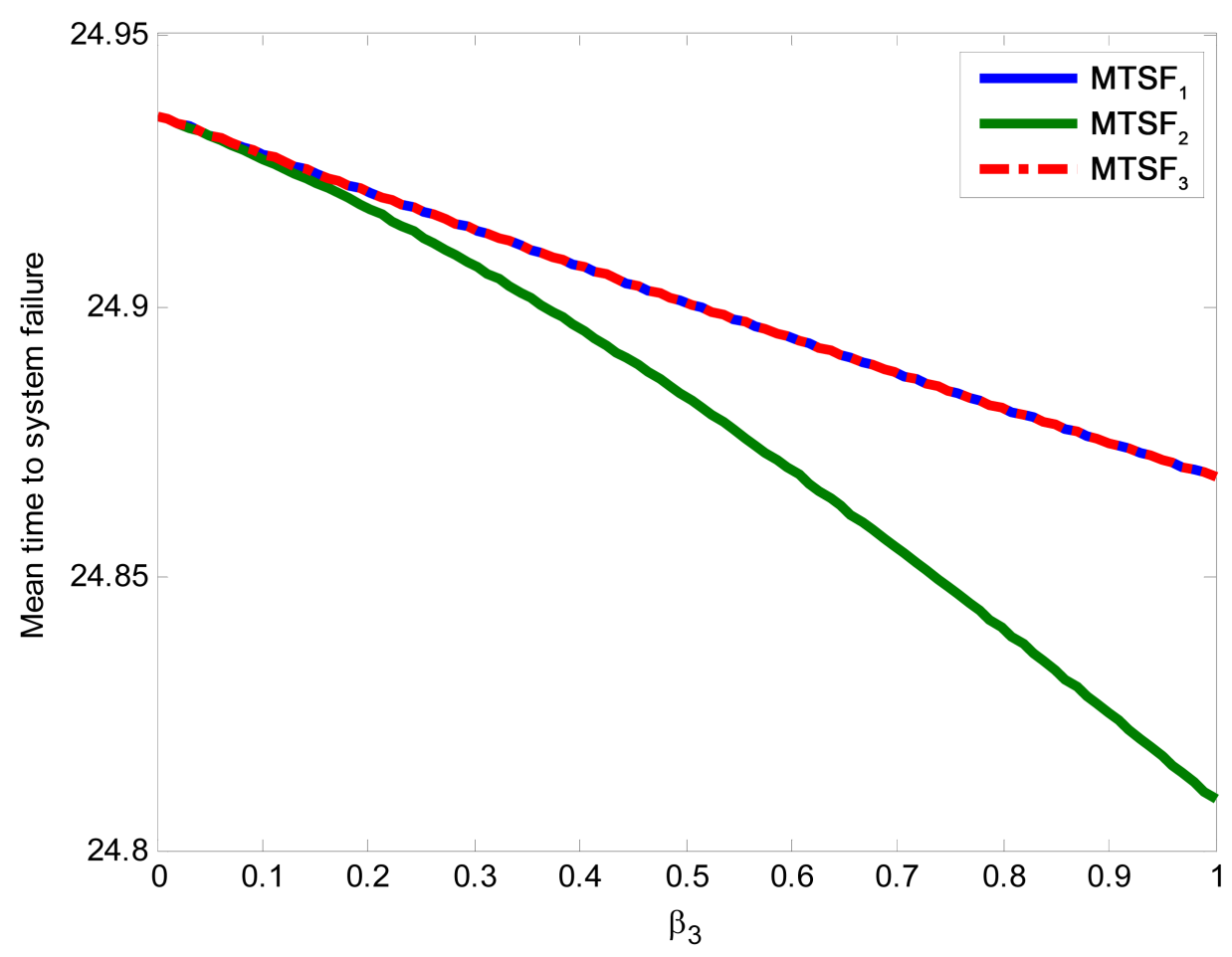

Figure 9. MTSF against $\beta_{3}$.

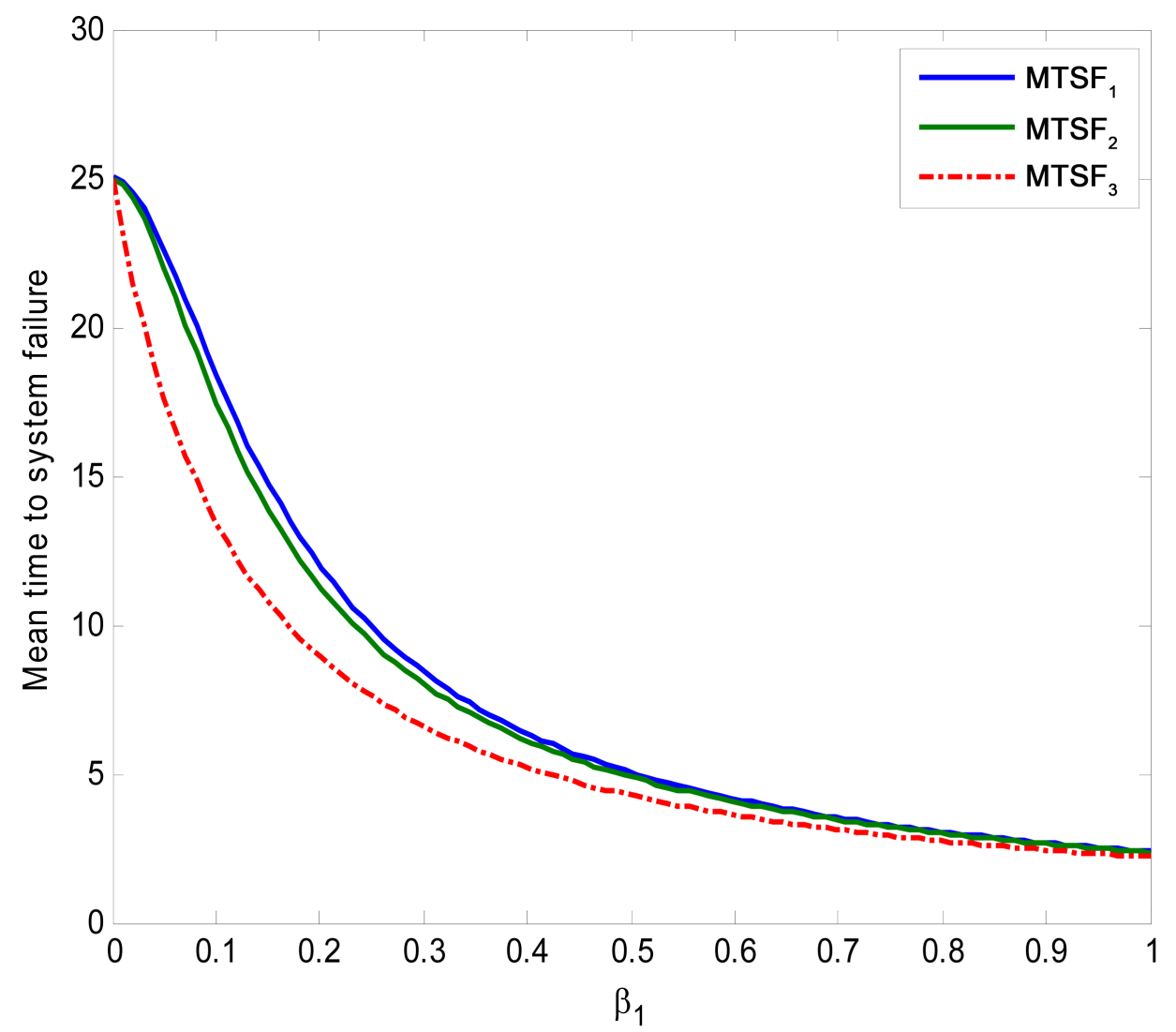

Figure 10. MTSF against $\beta_{1}$. 


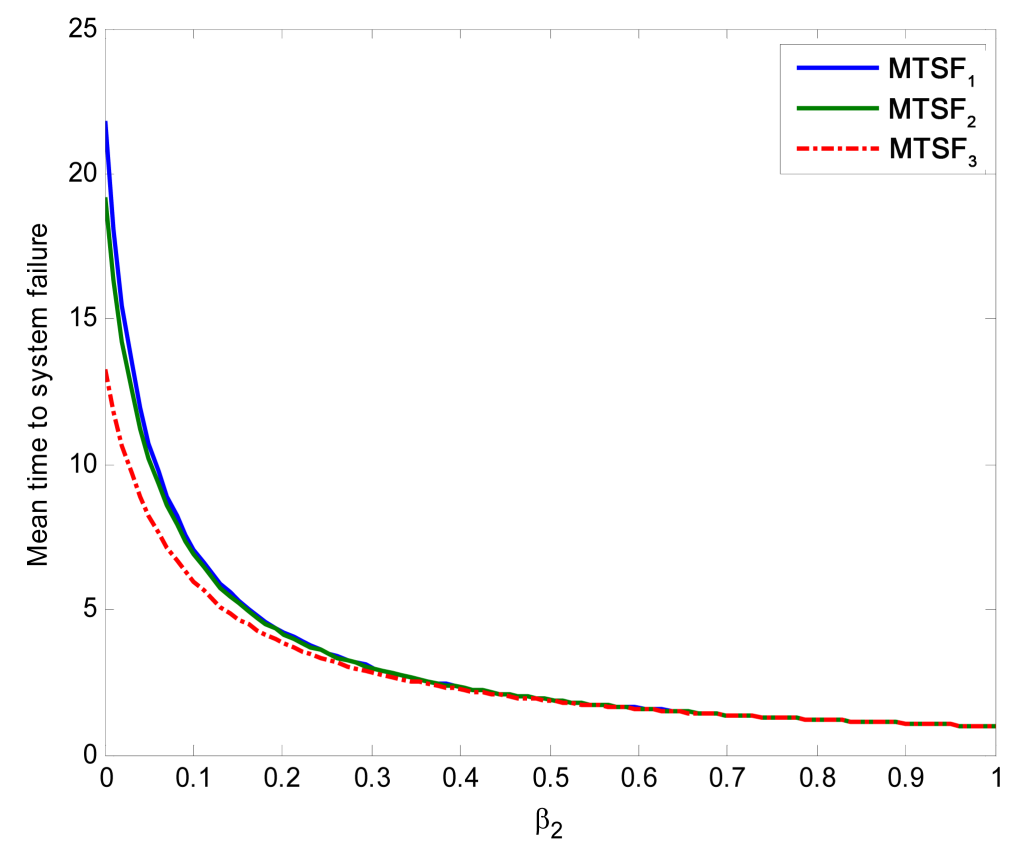

Figure 11. MTSF against $\beta_{2}$.

clear from the Figure that configuration II has higher availability with respect to $\alpha_{1}$ as compared with the other two configurations. There is slight difference between the availability of configuration II and that of configuration III with respect to $\alpha_{1}$. These tend to suggest that configuration II is better than the other configurations. Figure 2 depicts the availability calculations for the three configurations against $\beta_{1}$. The observations that can be made here are much similar to those made on Figure 1. From Figure 1 and Figure 2, it is clear that $A_{v 2}(\infty)>A_{v 3}(\infty)>A_{v 1}(\infty)$.

However, one can say that the results from Figure 3 show slight distinction between availability of three configurations with respect to $\alpha_{2}$. The differences between availability of configuration II and the other two configurations slightly increase as $\alpha_{2}$ increases. There is significant difference between the three configurations with respect to $\beta_{2}$ in Figure 4. It is evident from Figure 4 that configurations II and III have higher availability than configuration I as $\beta_{2}$ increases. Thus, $A_{v 2}(\infty)>A_{v 3}(\infty)>A_{v 1}(\infty)$ for Figure 3 and $A_{v 2}(\infty) \geq A_{v 3}(\infty)>A_{v 1}(\infty)$ for Figure 4 .

Results from Figure 5 and Figure 6 show slight distinction between availability of three configurations with respect to $\alpha_{3}$ and $\beta_{3}$. The differences between availability of configuration II and the other two configurations widen as $\alpha_{3}$ and $\beta_{3}$ increases respectively. It is evident from Figure 5 and Figure 6 that configurations II has higher availability than configuration I and III as $\alpha_{3}$ and $\beta_{2}$ increases. Thus, $A_{v 2}(\infty)>A_{v 3}(\infty)>A_{v 1}(\infty)$.

Simulations of MTSF for the three configurations depicted in Figures 7-9 show that MTSF increases as $\alpha_{1}$ and $\alpha_{3}$, and decreases as $\beta_{3}$ increases for any configuration. It is clear from these Figures that differences between MTSF of configuration I and III and configuration II widen as $\alpha_{1}, \alpha_{3}$ and $\beta_{3}$ increases respectively. It is evident from these Figures that configuration I and III have equal MTSF higher than configuration II as $\alpha_{1}$, $\alpha_{3}$ and $\beta_{3}$ increases. Figure 10 and Figure 11 show that the MTSF decreases as $\beta_{1}$ and $\beta_{2}$ for any configuration. It is evident from Figure 10 and Figure 11 that configurations I has higher MTSF than configuration II and III as $\beta_{1}$ and $\beta_{2}$ increases. Thus, from Figures 7-9, configuration I and II have equal MTSF. From Figure 10 the optimal configuration is configuration I while in Figure 11, configuration I and II have equal MTSF.

\section{Conclusion}

In this paper, we studied the reliability characteristics of three dissimilar systems connected supporting device. We developed the explicit expressions for steady-state availability and mean time to system failure (MTSF) for 
each configuration and performed comparative analysis numerically to determine the optimal configuration. It is evident from Figures 1-6 that configuration II is optimal configuration using steady-state availability while using MTSF, the optimal configuration depends on the values of $\alpha_{1}, \alpha_{3}, \beta_{1}, \beta_{2}$ and $\beta_{3}$. The present study will help the engineers and designers to develop sophisticated models and to design more critical system in interest of human kind.

\section{References}

[1] Khatab, A., Nahas, N. and Nourelfath, M. (2009) Availability of k-out-of-n: G Systems with Non Identical Components Subject to Repair Priorities. Reliability Engineering \& System Safety, 94, 142-151. http://dx.doi.org/10.1016/j.ress.2008.02.017

[2] Fathabadi, H.S. and Khodaei, M. (2012) Reliability Evaluation of Network Flows with Stochastic Capacity and Cost Constraint. International Journal of Mathematics in Operational Research, 4, 439-452. http://dx.doi.org/10.1504/IJMOR.2012.048904

[3] Khalili-Damghani, K. and Amiri, M. (2012) Solving Binary-State Multi-Objective Reliability Redundancy Allocation Seriesparallel Problem Using Efficient Epsilon Constraint, Multi-Start Partial Bound Enumeration Algorithm, and DEA. Reliability Engineering and System Safety, 103, 35-44. http://dx.doi.org/10.1016/j.ress.2012.03.006

[4] Khalili-Damghani, K., Abtahi, A.R., and Tavana, M. (2013) A New Multi-Objective Particle Swarm Optimization Method for Solving Reliability Redundancy Allocation Problems. Reliability Engineering and System Safety, 111, 5875. http://dx.doi.org/10.1016/j.ress.2012.10.009

[5] Wang, K.-H., Yen, T.-C. and Fang, Y.-C. (2012) Comparison of Availability between Two Systems with Warm Standby Units and Different Imperfect Coverage. Quality Technology and Quantitative Management, 9, 265-282

[6] Srinivasan, S.K. and Subramanian, R. (2006) Reliability Analysis of a Three Unit Warm Standby Redundant System with Repair. Annals of Operations Research, 143, 227-235. http://dx.doi.org/10.1007/s10479-006-7384-z

[7] Singh, V.V., Singh, B., Mangeyram and Goel, C.K. (2010) Availability Analysis of a System Having Three Units Super Priority, Priority and Ordinary under Peemmptive Resume Repair Policy. International Journal of Reliability and Applications, 11, 41-53.

[8] Singh, V.V. and Rawal, D.K. (2011) Availability Analysis of a System Having Two Units in Series Configuration with Controller and Human Failure under Different Repair Policies. International Journal of Scientific and Engineering Research, 2, 1-9.

[9] Yusuf, I. (2013) Comparison of Some Reliability Characteristics between Redundant Systems Requiring Supporting Units for Their Operation. Journal of Mathematical and Computational Sciences, 3, 216-232.

[10] Yusuf, I. (2014) Comparative Analysis of Profit between Three Dissimilar Repairable Redundant Systems Using Supporting External Device for Operation. Journal of Industrial Engineering International, 10, 2-9. http://dx.doi.org/10.1007/s40092-014-0077-3

[11] Wang, K.H. and Kuo, C.C. (2000) Cost and Probabilistic Analysis of Series Systems with Mixed Standby Components. Applied Mathematical Modelling, 24, 957-967. http://dx.doi.org/10.1016/S0307-904X(00)00028-7

[12] Wang, K., Hsieh, C. and Liou, C. (2006) Cost Benefit Analysis of Series Systems with Cold Standby Components and a Repairable Service Station. Journal of Quality Technology and Quantitative Management, 3, 77-92. 
Scientific Research Publishing (SCIRP) is one of the largest Open Access journal publishers. It is currently publishing more than 200 open access, online, peer-reviewed journals covering a wide range of academic disciplines. SCIRP serves the worldwide academic communities and contributes to the progress and application of science with its publication.

Other selected journals from SCIRP are listed as below. Submit your manuscript to us via either submit@scirp.org or Online Submission Portal.
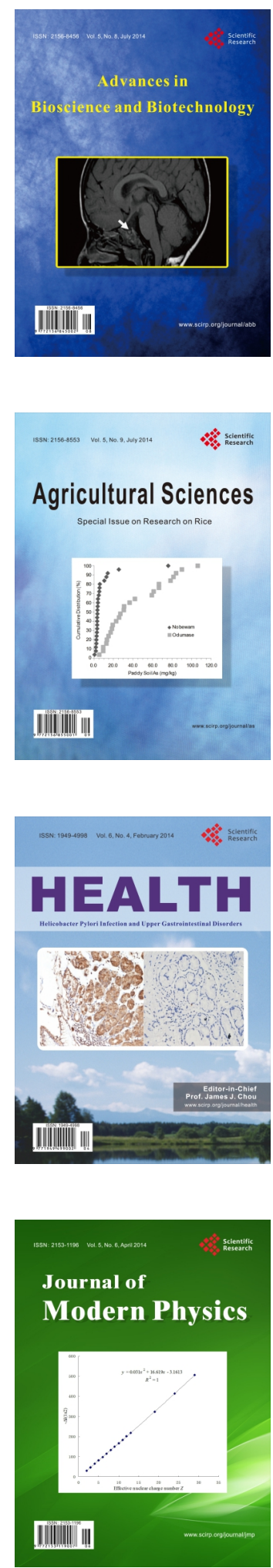
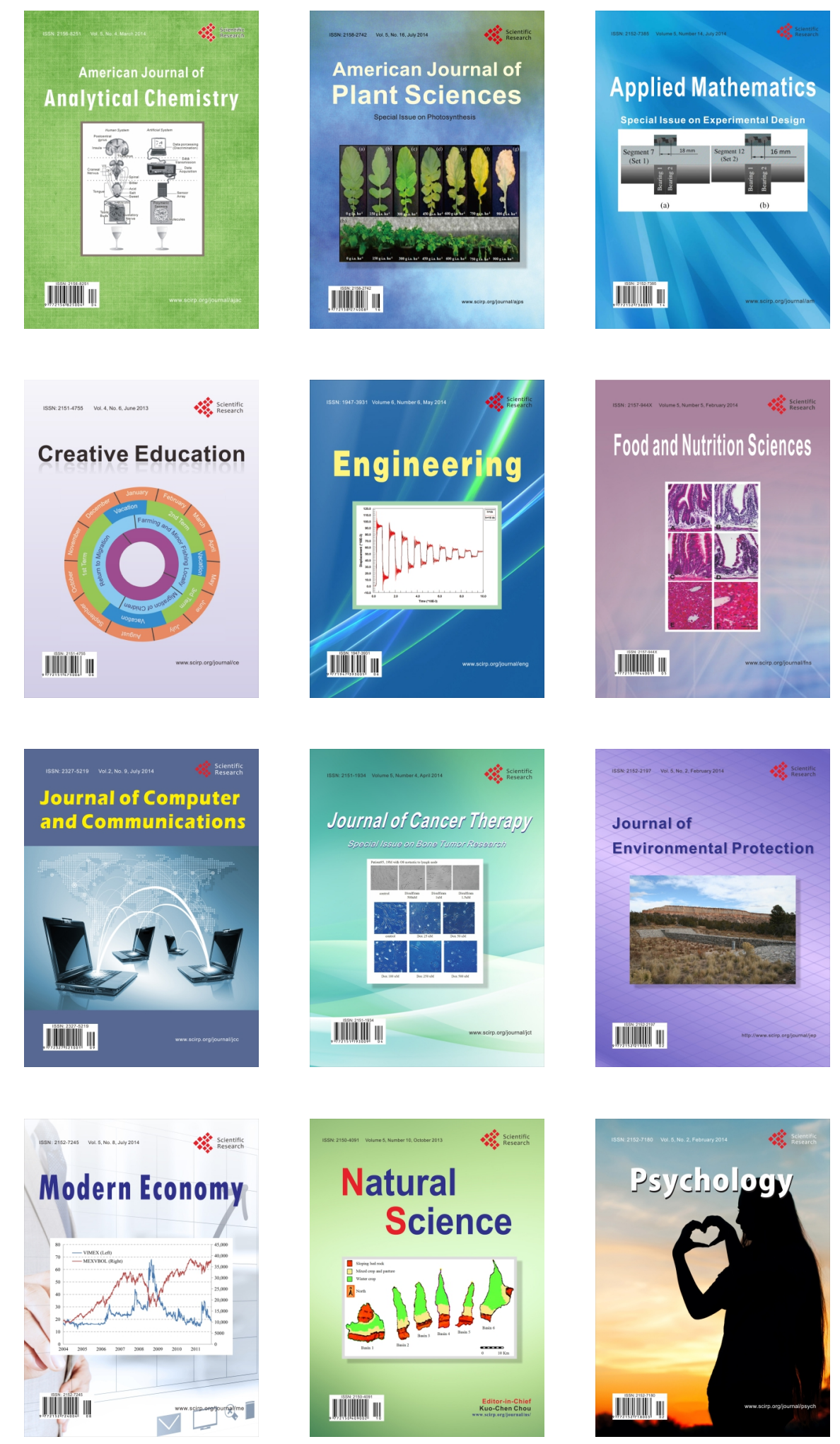